\title{
All congruences below stability-preserving fair testing or CFFD
}

\author{
Antti Valmari ${ }^{1}$ \\ Received: 24 April 2019 / Accepted: 30 December 2019 / Published online: 6 May 2020 \\ (c) The Author(s) 2020
}

\begin{abstract}
In process algebras, a congruence is an equivalence that remains valid when any subsystem is replaced by an equivalent one. Whether or not an equivalence is a congruence depends on the set of operators used in building systems from subsystems. Numerous congruences have been found, differing from each other in fine details, major ideas, or both, and none of them is good for all situations. The world of congruences seems thus chaotic, which is unpleasant, because the notion of congruence is at the heart of process algebras. This study continues attempts to clarify the big picture by proving that in certain sub-areas, there are no other congruences than those that are already known or found in the study. First, the region below stability-preserving fair testing equivalence is surveyed using an exceptionally small set of operators. The region contains few congruences, which is in sharp contrast with an earlier result on the region below Chaos-Free Failures Divergences (CFFD) equivalence, which contains 40 well-known and not well-known congruences. Second, steps are taken towards a general theory of dealing with initial stability, which is a small but popular detail. This theory is applied to the region below CFFD.
\end{abstract}

\section{Introduction}

This study is motivated by a striking difference: in the case of sequential computation, the notion of the result of a computation at the highest level of abstraction is simple, clear and widely agreed upon, whereas in the case of concurrent computation, many alternatives are widely used and numerous more alternatives are known to exist. Let us discuss this a bit.

It is universally agreed that a deterministic sequential program computes a partial function. The function is partial, because with some inputs the program may fail to terminate. This nice picture is slightly complicated by the fact that sequential programs may contain intentional nondeterminism, such as in the Miller-Rabin probabilistic primality test [1,13]; or unwanted

Congratulations to Rob van Glabbeek on his 60th birthday!.

Antti Valmari

antti.valmari@jyu.fi

1 Faculty of Information Technology, University of Jyväskylä, P.O. Box 35, 40014 University of Jyväskylä, Finland 
nondeterminism, such as in $i=i+++1$; (a Wikipedia example of undefined behavior). ${ }^{1}$ This issue could be taken into account by declaring that a sequential program executes a relation from the set of inputs to the set of outputs union $\{\perp\}:(i, o)$ is in the relation if and only if, for the input $i, o$ is a possible output or $o=\perp$ denoting failure to terminate. These abstract views to sequential programs are simple, natural, and widely accepted. At their level of abstraction, they have no rivals.

The situation is entirely different with concurrent programs. A concurrent program computes a behaviour. Behaviours may be — and have been — compared with branching bisimilarity [8], weak bisimilarity [10], CSP failures divergences equivalence [15], Chaos-Free Failures Divergences (CFFD) equivalence [20], and numerous other equivalences. None of them is widely considered as the "most natural" or "right" notion of "similar behaviour". If there is any agreement, it is that the choice of the most appropriate equivalence depends on the situation. Even the same users keep on switching between different equivalences depending on the task at hand, such as in [15], where, for instance, stable failures equivalence is used when the so-called catastrophic divergence phenomenon prevents the use of failures divergences equivalence.

The famous survey [5], among others, has improved our understanding a lot by presenting many equivalences in a systematic framework. However, such surveys do not provide full information, because they only discuss known equivalences. They leave it open whether there could be unknown useful equivalences with interesting properties.

In many situations the equivalence must be a congruence with respect to the operators that are available for building systems from subsystems. This requirement is so strong that it makes it possible to survey certain regions of equivalences, list all congruences in them, and prove that they contain no other congruences. Chapters 11 and 12 of [15] survey two regions and prove that there are three congruences in each. In [17], all congruences that are implied by the CFFD equivalence were found. This fairly large region contains 40 congruences, including stable failures equivalence, CSP failures divergences equivalence, and trace equivalence. Five kinds of failures, four kinds of infinite traces, two kinds of divergence traces, and two kinds of traces were needed, some of them new. Perhaps none of the previously unknown congruences among the 40 is interesting, but if so, then we know that no interesting congruences are lurking in that region.

A task that is somewhat similar in spirit to fully surveying a region is to choose a property such as deadlock-freedom and find the weakest congruence that preserves the property. Such results have been published in, e.g., [2,4,6,7,9,11,12,14,16]. As explained in [17], knowing the weakest congruence helps in designing compositional verification algorithms for the property.

The congruence property depends on the set of operators for building systems. Perhaps the most well known example of this deals with the common choice operator " + ". Branching bisimilarity, weak bisimilarity, CSP failures divergences equivalence, CFFD equivalence, and many other equivalences are not congruences with respect to it. In CSP, the congruence property was obtained by rejecting the common choice operator and introducing two other choice operators instead. In most other theories, the common choice operator was kept and the equivalence was refined so that it became a congruence.

At this point it is worth mentioning that if we are only interested in so-called safety properties of systems (that is, whatever the system does must be acceptable), then there is a single very widely agreed "right" congruence: trace equivalence. Furthermore, it was proven in

1 The $\mathrm{C}$ and $\mathrm{C}++$ specifications allow it to do just anything. In practice, the value of $i$ grows by either 1 or 2 . The assigned value $i+1$ is computed using the original value of $i$, but the assignment $=$ may be executed before or after the post-increment $i++$. 
[18] that any operator that satisfies a rather natural weak assumption can be constructed from parallel composition and hiding modulo trace equivalence, implying that trace equivalence is a congruence with respect to every "reasonable" operator. This situation is comparable to sequential programs in simplicity and clarity.

Things become problematic indeed, when also so-called liveness properties are of interest (the system must eventually do something useful, or at least not lose the ability to nondeterministically choose to eventually do something useful). The problems are so severe that they have led to wide adoption of an equivalence that does not imply trace equivalence, that is, CSP failures divergences equivalence.

The above-mentioned results in [15] use a fairly large set of operators. In particular, they use a "throw" operator that rules out many equivalences that would otherwise be congruences. The results in [17] only use parallel composition, hiding, relational renaming, and action prefix. Therefore, where the regions considered by [15] and [17] overlap, [17] gives additional congruences.

In [19], of which the present study is an extension, all congruences were found that are implied by the stability-preserving fair testing equivalence of [14]. This equivalence is a congruence. It is interesting for many reasons. It is the weakest congruence that preserves the property AG EF $a$, that is, "in all futures always, there is a future where eventually $a$ occurs". It offers an alternative approach to the verification of liveness properties. With the traditional approach, it is often necessary to explicitly state so-called fairness assumptions, which may be a burden. With fair testing equivalence this is unnecessary, because, so to speak, it has a built-in fairness assumption that is acceptable in many cases. Unlike other congruences for a significant subset of liveness properties, it has a very well-working partial order reduction method [21]. On the theoretical side, its definition is an interesting exception, because it seems somewhat ad-hoc instead of following a familiar pattern.

An important feature of [19] is that only parallel composition, hiding, and functional renaming were used for proving the absence of more congruences. This is a strictly smaller set of operators than in [15] and [17]. In [17] it was proven that if a congruence is implied by strong bisimilarity (this is a very weak assumption) and preserves anything, then it preserves at least the alphabet. It was shown with two counter-examples that the result depends on the availability of the action prefix and relational renaming operators. In [19], one of the counterexamples was encountered again, and six new (albeit uninteresting) congruences were found that do not preserve the alphabet.

The most important finding of [19] was that there is only one congruence between the not stability-preserving fair testing equivalence and the congruence that only preserves the alphabet: trace equivalence. If one wants to have something like fair testing, then one must go all the way to fair testing. There are no intermediate stops. This is in sharp contrast to [17]. It is also somewhat surprising, because the definition of fair testing seems quite ad-hoc, and because fair testing preserves AG EF $a$ which is a well-known example of a property that is not linear-time (e.g., [3, p. 32]). The importance of this result is strengthened by the fact that it was obtained in the presence of only parallel composition, hiding, and functional renaming. Also this is different from [17].

A widely used way to make an equivalence a congruence with respect to the common choice operator is to add information on initial stability: systems that can initially execute an invisible action are deemed inequivalent to systems that cannot. The study [19] was the first one that fully covers a region induced by a stability-preserving congruence. Also the weakest stability-preserving congruence was found. Some unexpected or at least unconventional congruences were found, but they may be considered uninteresting, because they rely on the absence of the action prefix operator. 
The present study makes two contributions. First, the conference paper [19] had a strict page limit, leading to dense proofs that are hard to read. The present study attempts to make the results in [19] more readable. Second, it develops a theory that greatly simplifies the treatment of initial stability when proving the absence of unknown congruences, at the cost of assuming the congruence property with respect to more operators than [19]. Therefore, it gives less general results on fair testing equivalence than [19]. On the other hand, it applies to CFFD equivalence.

Section 2 presents the necessary background concepts. The congruences that are implied by stability-preserving fair testing equivalence are introduced in Sect. 3. In Sect. 4, the weakest stability-preserving congruence is found. That stability-preserving fair testing equivalence does not imply more congruences is proven in Sect. 5. The new theory on adding initial stability checking is presented in Sect. 6, and applied to CFFD equivalence in Sect. 7 resulting in 79 congruences. This study is concluded by a discussion section.

\section{LTSs and their operators}

In this section we list many widely known concepts needed in this study, pointing out little facts that are useful to remember when reading our proofs. We also pay attention to details that vary in the literature, discussing the motivation of our choice.

The empty string is denoted with $\varepsilon$. The set of strings on $A$ is denoted with $A^{*}$, and $A^{+}=A^{*} \backslash\{\varepsilon\}$. If $\pi$ and $\sigma$ are strings, then $\pi \sqsubseteq \sigma$ denotes that $\pi$ is a prefix of $\sigma$, that is, there is a string $\rho$ such that $\sigma=\pi \rho$. If $\pi$ is a string and $K$ is a set of strings, then $\pi \sqsubseteq K$ denotes that there is $\sigma \in K$ such that $\pi \sqsubseteq \sigma$. We have $\varepsilon \sqsubseteq K$ if and only if $K \neq \emptyset$. We define $\pi^{-1} K=\{\rho \mid \pi \rho \in K\}$. It is nonempty if and only if $\pi \sqsubseteq K$. Trivially $\varepsilon^{-1} K=K$.

The invisible action is denoted with $\tau$. It denotes the occurrence of something that the outside world does not see. This is different from the occurrence of nothing, thus $\tau \neq \varepsilon$. An alphabet is any set $\Sigma$ such that $\varepsilon \notin \Sigma$ and $\tau \notin \Sigma$. Its elements are called visible actions.

A labelled transition system or LTS is a tuple $(S, \Sigma, \Delta, \hat{s})$ such that $\Sigma$ is an alphabet, $\Delta \subseteq S \times(\Sigma \cup\{\tau\}) \times S$, and $\hat{s} \in S$. Elements of $S$ and $\Delta$ are called states and transitions, respectively, and $\hat{s}$ is the initial state. The transition $\left(s, a, s^{\prime}\right)$ may also be denoted with $s-a \rightarrow s^{\prime}$. By $s-a \rightarrow$ we mean that there is $s^{\prime}$ such that $s-a \rightarrow s^{\prime}$.

If an LTS is shown as a drawing, then, unless otherwise stated, its alphabet is the set of the visible actions along the transitions in the drawing. The alphabet may be specified explicitly in the text or near the bottom right corner of the drawing. For instance, the alphabet of $\circ \stackrel{\tau}{\longrightarrow} \stackrel{a}{\longrightarrow} \circ$ is $\{a\}$ and the alphabet of $\circ \stackrel{\tau}{\longrightarrow} \stackrel{a}{\longrightarrow} 0\{a, b\}$ is $\{a, b\}$. In particular, we will frequently use $O$ and $\sigma \tau$, their alphabets being $\emptyset$.

In the constructions of this study, we will often need elements that are not in a given alphabet or in a given set of states. Such entities exist because, by the axiom of foundation in set theory, if $X$ is a set, then $X,\{X\},\{\{X\}\}$, and so on are not elements of $X$. Sometimes in the literature, instead of each LTS having an alphabet of its own, there is a single global alphabet. That convention would make things difficult in the present study, because elements that are not in the alphabet would not be available. We will return to this issue in Sect. 8.

We use $L, M, L^{\prime}, M^{\prime}, L_{1}, M_{1}$, and so on to denote LTSs. Unless otherwise stated, $L=(S, \Sigma, \Delta, \hat{s}), L^{\prime}=\left(S^{\prime}, \Sigma^{\prime}, \Delta^{\prime}, \hat{s}^{\prime}\right), L_{1}=\left(S_{1}, \Sigma_{1}, \Delta_{1}, \hat{s}_{1}\right)$, and so on. Because this convention is sometimes unclear, we also use $\Sigma(L)$ to denote the alphabet of $L$. By $s-a \rightarrow_{i} s^{\prime}$ we mean that $\left(s, a, s^{\prime}\right) \in \Delta_{i}$. 
Researchers widely agree that at the detailed level, it is appropriate to compare behaviours using the following notion. Two LTSs $L_{1}$ and $L_{2}$ are bisimilar, denoted with $L_{1} \equiv L_{2}$, if and only if $\Sigma_{1}=\Sigma_{2}$ and there is a relation " “ " $\subseteq S_{1} \times S_{2}$ with the following properties:

1. $\hat{s}_{1} \sim \hat{s}_{2}$.

2. If $s_{1}-a \rightarrow_{1} s_{1}^{\prime}$ and $s_{1} \sim s_{2}$, then there is $s_{2}^{\prime}$ such that $s_{2}-a \rightarrow_{2} s_{2}^{\prime}$ and $s_{1}^{\prime} \sim s_{2}^{\prime}$.

3. If $s_{2}-a \rightarrow_{2} s_{2}^{\prime}$ and $s_{1} \sim s_{2}$, then there is $s_{1}^{\prime}$ such that $s_{1}-a \rightarrow_{1} s_{1}^{\prime}$ and $s_{1}^{\prime} \sim s_{2}^{\prime}$.

It is easy to check that if $L_{1}$ and $L_{2}$ are isomorphic, then they are bisimilar.

The reachable part of an LTS $(S, \Sigma, \Delta, \hat{s})$ is $\left(S^{\prime}, \Sigma, \Delta^{\prime}, \hat{s}\right)$, where $S^{\prime}$ and $\Delta^{\prime}$ consist of those states and transitions to which there is a path from $\hat{s}$. Any LTS is bisimilar with its reachable part.

Next we define the six operators that this study will focus on.

Parallel composition $L_{1} \| L_{2}$ It is the reachable part of $(S, \Sigma, \Delta, \hat{s})$, where $S=S_{1} \times S_{2}$, $\Sigma=\Sigma_{1} \cup \Sigma_{2}, \hat{s}=\left(\hat{s}_{1}, \hat{s}_{2}\right)$, and $\left(s_{1}, s_{2}\right)-a \rightarrow\left(s_{1}^{\prime}, s_{2}^{\prime}\right)$ if and only if

$-a \notin \Sigma_{2}, s_{1}-a \rightarrow_{1} s_{1}^{\prime}$, and $s_{2}^{\prime}=s_{2} \in S_{2}$,

- $a \notin \Sigma_{1}, s_{2}-a \rightarrow_{2} s_{2}^{\prime}$, and $s_{1}^{\prime}=s_{1} \in S_{1}$, or

$-a \in \Sigma_{1} \cap \Sigma_{2}, s_{1}-a \rightarrow_{1} s_{1}^{\prime}$, and $s_{2}-a \rightarrow_{2} s_{2}^{\prime}$.

That is, if $a$ belongs to the alphabets of both components, then an $a$-transition of the parallel composition consists of simultaneous $a$-transitions of both components. If $a$ belongs to the alphabet of one but not the other component, then that component may make an $a$-transition while the other component stays in its current state. Also each $\tau$-transition of the parallel composition consists of one component making a $\tau$-transition without the other participating. The result of the parallel composition is pruned by only taking the reachable part.

It is easy to check that $L_{1} \| L_{2}$ is isomorphic to (and thus bisimilar with) $L_{2} \| L_{1}$, and $\left(L_{1} \| L_{2}\right) \| L_{3}$ is isomorphic to $L_{1} \|\left(L_{2} \| L_{3}\right)$. This means that "\|" can be considered commutative and associative.

Hiding $L \backslash A$ Let $A$ be a set. The hiding of $A$ in $L$ is $\left(S, \Sigma^{\prime}, \Delta^{\prime}, \hat{s}\right)$, where $\Sigma^{\prime}=\Sigma \backslash A$ and $\Delta^{\prime}=\left\{\left(s, a, s^{\prime}\right) \in \Delta \mid a \notin A\right\} \cup\left\{\left(s, \tau, s^{\prime}\right) \mid \exists a \in A:\left(s, a, s^{\prime}\right) \in \Delta\right\}$. That is, labels of transitions that are in $A$ are replaced by $\tau$ and removed from the alphabet. Other labels of transitions are not affected.

Relational renaming $L \Phi$ Let $\Phi$ be a set of pairs such that for every $(a, b) \in \Phi$ we have $\tau \neq a \neq \varepsilon$ and $\tau \neq b \neq \varepsilon$. The domain of $\Phi$ is $\mathcal{D}(\Phi)=\{a \mid \exists b:(a, b) \in \Phi\}$. Let the predicate $\Phi(a, b)$ hold if and only if either $(a, b) \in \Phi$ or $b=a \notin \mathcal{D}(\Phi)$. The relational renaming of $L$ with $\Phi$ is $\left(S, \Sigma^{\prime}, \Delta^{\prime}, \hat{s}\right)$, where $\Sigma^{\prime}=\{b \mid \exists a \in \Sigma: \Phi(a, b)\}$ and $\Delta^{\prime}=\left\{\left(s, b, s^{\prime}\right) \mid \exists a:\left(s, a, s^{\prime}\right) \in \Delta \wedge \Phi(a, b)\right\}$.

That is, $\Phi$ renames visible actions to visible actions. A visible action may be renamed to more than one visible action. In that case, the transitions labelled by that action are duplicated as needed. If $\Phi$ specifies no new names for an action, the transitions labelled by it remain unchanged. In particular, $\tau$-transitions remain unchanged. The alphabet of the result consists of the new names of the original visible actions where such have been defined, and of the remaining original visible actions as such. Pairs in $\Phi$ whose first component is not in $\Sigma$ have no effect. This design makes it simple to specify the intended changes without causing accidental removal of the transitions that are not intended to change.

\footnotetext{
2 When a relation symbol is used as a relation, it returns a truth value and must be between an element of the domain and an element of the codomain. When a relation symbol is used as a set of pairs, usually neither of these holds. This significantly affects the intended parsing and interpretation of the expression. To reduce the risk of mis-interpretation, the author tends to point out uses as a set of pairs with double quotes.
} 
Functional renaming $\phi(L)$ Functional renaming is the subcase of relational renaming where $\Phi$ specifies at most one new action name for each action. It is denoted with $\phi(L)$, where $\phi(a)=b$ if $(a, b) \in \Phi$, and $\phi(a)=a$, otherwise. It is included in our list of six operators, because we will encounter some equivalences that are congruences with respect to it but not with respect to relational renaming.

We will frequently use the following two special cases of functional renaming as helpful notation in proofs. They attach and remove an integer $i$ to visible actions. They will make it easy to ensure that in a parallel composition, precisely those actions synchronize whom we want to synchronize. In the notation, $A$ is an alphabet, $\varepsilon \neq a \neq \tau$, and $\varepsilon \neq a_{j} \neq \tau$ for $1 \leq j \leq n$. Without loss of generality we assume that always $\varepsilon \neq a^{[i]} \neq \tau$.

$$
\begin{aligned}
a^{[i]} & :=(a, i) \\
\left(a_{1} a_{2} \cdots a_{n}\right)^{[i]} & :=a_{1}^{[i]} a_{2}^{[i]} \cdots a_{n}^{[i]} \\
A^{[i]} & :=\left\{a^{[i]} \mid a \in A\right\} \\
\lceil L\rceil^{[i]} & :=L \Phi, \text { where } \Phi=\left\{\left(a, a^{[i]}\right) \mid a \in \Sigma\right\} \\
\lfloor L\rfloor_{[i]} & :=L \Phi, \text { where } \Phi=\left\{\left(a^{[i]}, a\right) \mid a^{[i]} \in \Sigma\right\}
\end{aligned}
$$

Action prefix a.L. Let $a \neq \varepsilon$. Let $\Sigma^{\prime}=\Sigma \cup\{a\}$ if $a \neq \tau$, and $\Sigma^{\prime}=\Sigma$ otherwise. The operator $a . L$ yields $\left(S^{\prime}, \Sigma^{\prime}, \Delta^{\prime}, \hat{s}^{\prime}\right)$, where $\hat{s}^{\prime}$ is a new state (that is, $\hat{s}^{\prime} \notin S$ ), $S^{\prime}=S \cup\left\{\hat{s}^{\prime}\right\}$, and $\Delta^{\prime}=\Delta \cup\left\{\left(\hat{s}^{\prime}, a, \hat{s}\right)\right\}$. That is, $a . L$ starts by executing $a$, after which it is in the initial state of $L$.

Choice $L_{1}+L_{2}$ Roughly speaking, the choice between $L_{1}$ and $L_{2}$ starts by executing an initial transition of $L_{1}$ or an initial transition of $L_{2}$. This transition represents a choice between $L_{1}$ and $L_{2}$. Then $L_{1}+L_{2}$ continues like the chosen LTS continues after the corresponding transition.

This may be formalized by taking a disjoint union of $L_{1}$ and $L_{2}$, and adding a new state that acts as the initial state of the result. For each initial transition of $L_{1}$ and of $L_{2}$, a copy is made that starts at the new state. Indexing of state names is used to ensure that the union is disjoint. That is, $L_{1}+L_{2}=\left(S^{\prime}, \Sigma^{\prime}, \Delta^{\prime}, \hat{s}^{\prime}\right)$, where $S^{\prime}=S_{1}^{[1]} \cup S_{2}^{[2]} \cup\left\{\hat{s}^{\prime}\right\}, \Sigma^{\prime}=\Sigma_{1} \cup \Sigma_{2}$, $\Delta^{\prime}=\Delta_{1}^{\prime} \cup \Delta_{1}^{\prime \prime} \cup \Delta_{2}^{\prime} \cup \Delta_{2}^{\prime \prime}$, and $\hat{s}^{\prime} \notin S_{1}^{[1]} \cup S_{2}^{[2]}$, where $\Delta_{i}^{\prime}=\left\{\left(s^{[i]}, a, s^{\prime[i]}\right) \mid\left(s, a, s^{\prime}\right) \in \Delta_{i}\right\}$ and $\Delta_{i}^{\prime \prime}=\left\{\left(\hat{s}^{\prime}, a, s^{\prime[i]}\right) \mid\left(\hat{s}_{i}, a, s^{\prime}\right) \in \Delta_{i}\right\}$ for $i \in\{1,2\}$.

Also "+" can be considered commutative and associative (up to bisimilarity).

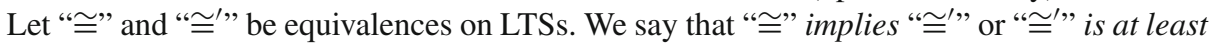

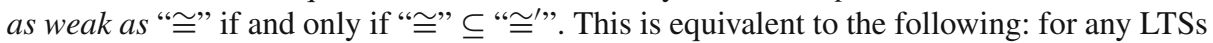
$L_{1}$ and $L_{2}$ we have $L_{1} \cong L_{2} \Rightarrow L_{1} \cong L_{2}$.

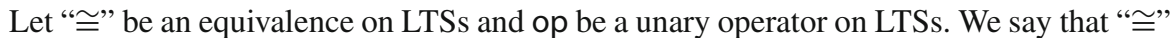
is a congruence with respect to op if and only if for every $L$ and $L^{\prime}, L \cong L^{\prime} \operatorname{implies}$ op $(L) \cong$ op $\left(L^{\prime}\right)$. When we say that an equivalence is a congruence with respect to parallel composition, we mean that it is a congruence with respect to the two unary operators $\operatorname{op}_{1}(L):=L_{1} \| L$ and $\mathrm{op}_{2}(L):=L \| L_{2}$. Because " $\|$ " is commutative, this is equivalent to saying that the equivalence is a congruence with respect to op ${ }_{1}(L)$. The similar convention and remark apply to "+".

It is easy to show with induction that if $f\left(L_{1}, \ldots, L_{n}\right)$ is an expression, $L_{i} \cong L_{i}^{\prime}$ for $1 \leq$

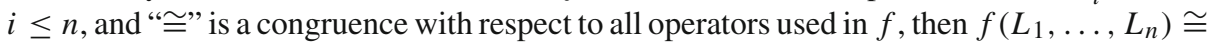
$f\left(L_{1}^{\prime}, \ldots, L_{n}^{\prime}\right)$.

\section{Stability-preserving fair testing and the region below it}

In this section we define 4 times 5 equivalences in a two-dimensional fashion. Stabilitypreserving fair testing equivalence is the strongest equivalence among them. We prove that 
17 of these equivalences are congruences with respect to parallel composition, hiding, and functional renaming. We investigate the congruence properties of these 17 also with respect to relational renaming, action prefix, and choice. We will see that the remaining three equivalences are not congruences with respect to parallel composition.

An LTS $L$ is unstable if and only if $\hat{s}-\tau \rightarrow$, and stable otherwise. If $L$ is stable we define en $(L):=\{a \in \Sigma \mid \hat{s}-a \rightarrow\}$, that is, the set of visible actions that $L$ can execute in its initial state. If $L$ is unstable, then the value of en $(L)$ is not important. By defining it as en $(L):=\{\tau\}$ we get the handy property that if $L$ is stable and $L^{\prime}$ is unstable, then certainly en $(L) \neq$ en $\left(L^{\prime}\right)$. The following lemma tells how stability and en behave in LTS expressions.

Lemma $1-L_{1} \| L_{2}$ is stable if and only if both $L_{1}$ and $L_{2}$ are stable.

Then en $\left(L_{1} \| L_{2}\right)=\left(e n\left(L_{1}\right) \backslash \Sigma_{2}\right) \cup\left(e n\left(L_{2}\right) \backslash \Sigma_{1}\right) \cup\left(e n\left(L_{1}\right) \cap e n\left(L_{2}\right)\right)$.

- $L \backslash A$ is stable if and only if $L$ is stable and en $(L) \cap A=\emptyset$.

Then en $(L \backslash A)=$ en $(L)$.

- $L \Phi$ is stable if and only if $L$ is stable.

Then en $(\Phi(L))=\{b \mid \exists a \in \operatorname{en}(L): \Phi(a, b)\}$.

$-\phi(L)$ is stable if and only if $L$ is stable.

Then en $(\phi(L))=\{\phi(a) \mid a \in$ en $(L)\}$.

- a.L is stable if and only if $a \neq \tau$.

Then en $(a . L)=\{a\}$.

$-L_{1}+L_{2}$ is stable if and only if both $L_{1}$ and $L_{2}$ are stable.

Then en $\left(L_{1}+L_{2}\right)=e n\left(L_{1}\right) \cup$ en $\left(L_{2}\right)$.

If $s \in S, s^{\prime} \in S$, and $\sigma \in \Sigma^{*}$, then $s=\sigma \Rightarrow s^{\prime}$ denotes that $L$ contains a path from $s$ to $s^{\prime}$ such that the sequence of visible actions along it is $\sigma$. In particular, $s=\varepsilon \Rightarrow s$ holds for every $s \in S$. The notation $s=\sigma \Rightarrow$ means that there is $s^{\prime}$ such that $s=\sigma \Rightarrow s^{\prime}$. The set of traces of $L$ is $\operatorname{Tr}(L):=\{\sigma \mid \hat{s}=\sigma \Rightarrow\}$. If $L$ is stable, then en $(L)=\operatorname{Tr}(L) \cap \Sigma$.

A state $s$ of $L$ refuses the string $\rho$ if and only if $s=\rho \Rightarrow$ does not hold. That is, refusing a string means inability to execute it to completion. Refusing a set means refusing its every element. A tree failure of $L$ is a pair $(\sigma, K)$ where $\sigma \in \Sigma^{*}$ and $K \subseteq \Sigma^{+}$such that there is $s$ such that $\hat{s}=\sigma \Rightarrow s$ and $s$ refuses $K$ [14]. The empty string $\varepsilon$ is ruled out from $K$ because $s=\varepsilon \Rightarrow$ holds for every state $s$. In the failures of CSP [15] or CFFD [17], $K$ is a set of visible actions, while now it is a set of strings of visible actions.

The set of the tree failures of $L$ is denoted with $\operatorname{Tf}(L)$. The following lemmas express simple properties of tree failures that will be used in the sequel.

Lemma 2 1. If $\Sigma=\emptyset$, then $\operatorname{Tr}(L)=\{\varepsilon\}$ and $\operatorname{Tf}(L)=\{(\varepsilon, \emptyset)\}$.

2. If $\sigma \in \operatorname{Tr}(L)$, then $(\sigma, \emptyset) \in \operatorname{Tf}(L)$.

3. If $\sigma \notin \operatorname{Tr}(L)$, then, for every $\pi$ and $K,(\sigma \pi, K) \notin \operatorname{Tf}(L)$.

Proof The first two claims are immediate from the definitions. The third claim follows from the fact that if $\sigma \notin \operatorname{Tr}(L)$, then $\sigma \pi \notin \operatorname{Tr}(L)$.

Lemma 3 Assume that $\hat{s}=\sigma \Rightarrow s$ and, for every $a \in \Sigma, \neg(s=a \Rightarrow)$. Then $(\sigma, K) \in \operatorname{Tf}(L)$ if and only if $K \subseteq \Sigma^{+}$.

Proof It is immediate from the definition that if $(\sigma, K) \in \operatorname{Tf}(L)$, then $K \subseteq \Sigma^{+}$. If $K \subseteq \Sigma^{+}$, the state $s$ guarantees that $(\sigma, K) \in \operatorname{Tf}(L)$ by blocking the first action of every element in $K$. 
In particular, $\operatorname{Tf}(\delta ص \tau)=\operatorname{Tf}(\delta)$, implying $\operatorname{Tf}\left(L \|^{\prime} \circlearrowright \tau\right)=\operatorname{Tf}(L)$. This is a major difference between tree failures and the failures in CSP or CFFD theories. In CSP failures divergences equivalence divergence is catastrophic [15], meaning, among other things, that for every $L$

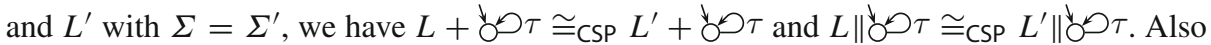
CFFD equivalence is sensitive to divergence, but in a much less dramatic fashion [17]. We mention already now that fair testing equivalence is insensitive to divergence.

Lemma 4 Assume that $L$ is stable and $K \subseteq \Sigma^{+}$. We have $(\varepsilon, K) \in \operatorname{Tf}(L)$ if and only if $K \cap \operatorname{Tr}(L)=\emptyset$.

Proof Because $L$ is stable, $\hat{s}=\varepsilon \Rightarrow s$ implies $s=\hat{s}$. Therefore, $(\varepsilon, K) \in \operatorname{Tf}(L)$ if and only if $\hat{s}$ refuses $K$. Furthermore, $\hat{s}$ refuses $\rho$ if and only if $\rho \notin \operatorname{Tr}(L)$.

The notation $L_{1} \preceq L_{2}$ denotes that for every $(\sigma, K) \in \operatorname{Tf}\left(L_{1}\right)$, either $(\sigma, K) \in \operatorname{Tf}\left(L_{2}\right)$ or there is $\pi$ such that $\pi \sqsubseteq K$ and $\left(\sigma \pi, \pi^{-1} K\right) \in \operatorname{Tf}\left(L_{2}\right)$. The latter condition is motivated by the following example. If $L=\circ \stackrel{a}{\leftrightarrow} \stackrel{a}{\rightarrow} \rightarrow \circ \stackrel{a}{\rightarrow}$, then $(\varepsilon,\{a a\}) \notin \operatorname{Tf}(L)$. Even so, $\left(L \| a . a . b .{ }^{\natural}\right) \backslash\{a\}$ may fail to execute $b$. Here $\left(\sigma \pi, \pi^{-1} K\right) \in \operatorname{Tf}(L)$, where $\sigma=\varepsilon, \pi=a$, and $\pi^{-1} K=\{a\}$. For a more detailed discussion, please see [14].

The condition $(\sigma, K) \in \operatorname{Tf}\left(L_{2}\right)$ is only needed to deal with the case $K=\emptyset$, because when $K \neq \emptyset$ it is obtained from the latter condition by choosing $\pi=\varepsilon$. The LTSs $L_{1}$ and $L_{2}$ are fair testing equivalent, if and only if $\Sigma_{1}=\Sigma_{2}, L_{1} \preceq L_{2}$, and $L_{2} \preceq L_{1}$ [14].

If $A$ and $B$ are sets, let $A \# B:=(A \backslash B) \cup(B \backslash A)$.

Lemma 5 The following relation is an equivalence on sets: $A \approx B$ if and only if $A \# B$ is finite.

Proof Because $A \# A=\emptyset$, “ $\approx$ " is reflexive. Because $A \# B=B \# A$, \# $\approx$ " is symmetric. To prove transitivity, assume that $A \approx B$ and $B \approx C$. That is, $A \# B$ and $B \# C$ are finite. If $a \in A \backslash C$, then $a \in A \backslash B$ or $a \in B \backslash C$. So $A \backslash C \subseteq(A \backslash B) \cup(B \backslash C)$. A symmetric claim holds if $a \in C \backslash A$. Thus $A \# C \subseteq(A \# B) \cup(B \# C)$. Therefore, also $A \# C$ is finite, that is, $A \approx C$.

Lemma 6 Let $f_{1}(L), \ldots, f_{n}(L)$ be functions from LTSs to some sets $D_{1}, \ldots, D_{n}$, and let " $\approx_{i}$ " be equivalences on $D_{i}$ for $1 \leq i \leq n$. Assume that " $\cong$ " has been defined via $L \cong L^{\prime}$

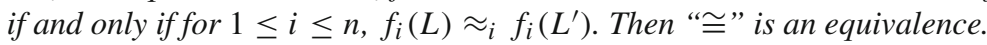

Proof For any $L$ and for $1 \leq i \leq n, f_{i}(L) \approx_{i} f_{i}(L)$, because " $\approx_{i}$ " is reflexive. Therefore,

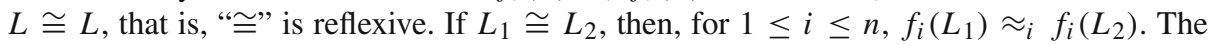

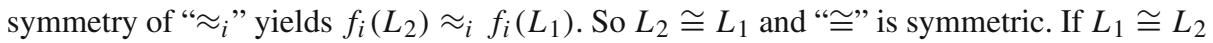
and $L_{2} \cong L_{3}$, then, for $1 \leq i \leq n, f_{i}\left(L_{1}\right) \approx_{i} f_{i}\left(L_{2}\right) \approx_{i} f_{i}\left(L_{3}\right)$, yielding $f_{i}\left(L_{1}\right) \approx_{i} f_{i}\left(L_{3}\right)$ by the transitivity of " $\approx_{i}$ ". This means $L_{1} \cong L_{3}$. Therefore, "ㅇ" is transitive.

We now define a number of equivalences, of which twenty will be discussed in detail. The twenty will be shown in Fig. 1. Five of them do not preserve initial stability. The remaining 15 are defined by using one of the five to compare unstable LTSs, one of three equivalences to compare stable LTSs, and declaring that a stable and an unstable LTS are never equivalent. Eight of these 20 equivalences do not preserve the alphabet. If they were not congruences, they would be uninteresting indeed. However, they are congruences, and thus serve as examples of oddities that may be found when studying all congruences. The reader can skip them by skipping everything that contains \# or $\perp$. 
Definition 7 Let $L_{1}$ and $L_{2}$ be LTSs, and let $\{x, y\} \subseteq\{\perp, \#, \Sigma$, en, $\operatorname{tr}$, ft $\}$. We define

- $L_{1} \cong{ }_{\perp} L_{2}$ holds for every $L_{1}$ and $L_{2}$,

- $L_{1} \cong_{\#} L_{2}$ if and only if $\Sigma_{1} \# \Sigma_{2}$ is finite,

- $L_{1} \cong \Sigma L_{2}$ if and only if $\Sigma_{1}=\Sigma_{2}$,

- $L_{1} \cong_{\text {en }} L_{2}$ if and only if $\Sigma_{1}=\Sigma_{2}$ and en $\left(L_{1}\right)=\operatorname{en}\left(L_{2}\right)$ (this one will not be used on unstable LTSs),

- $L_{1} \cong_{\operatorname{tr}} L_{2}$ if and only if $\Sigma_{1}=\Sigma_{2}$ and $\operatorname{Tr}\left(L_{1}\right)=\operatorname{Tr}\left(L_{2}\right)$ (trace equivalence),

- $L_{1} \cong_{\mathrm{ft}} L_{2}$ if and only if $\Sigma_{1}=\Sigma_{2}, L_{1} \preceq L_{2}$, and $L_{2} \preceq L_{1}$ (fair testing equivalence), and

- $L_{1} \cong_{y}^{x} L_{2}$ if and only if

- $L_{1} \cong_{x} L_{2}$ and $L_{1}$ and $L_{2}$ are both stable, or

- $L_{1} \cong{ }_{y} L_{2}$ and $L_{1}$ and $L_{2}$ are both unstable (stability-preserving equivalences).

For instance, " $\cong \mathrm{ft}$ " compares stable LTSs with fair testing equivalence and unstable LTSs with trace equivalence. It also preserves initial stability, that is, $L_{1} \cong \mathrm{ft}$ The relation " $\cong \sum$ ", equates two LTSs if and only if they have the same alphabet and either both or none of them is stable.

Lemma 8 If " $\cong x$ " is an equivalence on stable LTSs and " $\cong_{y}$ " is an equivalence on unstable

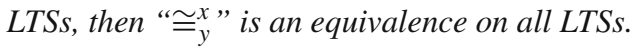

Proof If $L$ is stable then $L \cong_{x} L$ holds and yields $L \cong_{y}^{x} L$. Otherwise $L \cong_{y} L$ holds and yields $L \cong{ }_{y}^{x} L$.

Assume $L_{1} \cong_{y}^{x} L_{2}$. If $L_{1}$ is stable, then $L_{2}$ is as well and $L_{1} \cong_{x} L_{2}$. It implies $L_{2} \cong_{x} L_{1}$ and $L_{2} \cong{ }_{y}^{x} L_{1}$. If $L_{1}$ is unstable, similar reasoning applies with " $\cong y$ ".

To prove that " $\cong x$ " is transitive, let $L_{1} \cong_{y}^{x} L_{2}$ and $L_{2} \cong_{y}^{x} L_{3}$. If $L_{2}$ is stable, then $L_{1}$ is as well by $L_{1} \cong_{y}^{x} L_{2}$, and $L_{3}$ by $L_{2} \cong_{y}^{x} L_{3}$. So $L_{1} \cong_{x} L_{2} \cong_{x} L_{3}$, yielding $L_{1} \cong_{x} L_{3}$ and $L_{1} \cong{ }_{y}^{x} L_{3}$. Similar reasoning applies if $L_{2}$ is unstable.

\section{Lemma 9 The relations in Definition 7 are equivalences.}

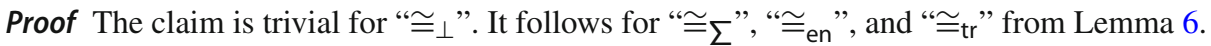

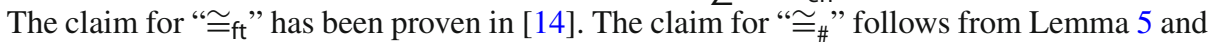
Lemma 6, and the remaining claim from Lemma 8.

The 17 equivalences that will be proven congruences are shown in Fig. 1, together with three equivalences that arise from Definition 7 but are not congruences. There is a path downwards from an equivalence to an equivalence in the figure if and only if the former implies the latter. This holds because of the following. In [14] it was shown that " $\cong_{\mathrm{ft}}$ " implies " $\cong \mathrm{tr}$ ". The same follows easily from Lemma 2(2) and (3). Clearly $L_{1} \cong \operatorname{tr} L_{2} \Rightarrow L_{1} \cong \Sigma L_{2} \Rightarrow L_{1} \cong_{\#}$ $L_{2} \Rightarrow L_{1} \cong_{\perp} L_{2}$ and $L_{1} \cong_{\text {en }} L_{2} \Rightarrow L_{1} \cong{ }_{\Sigma} L_{2}$. Furthermore, if $L_{1}$ and $L_{2}$ are stable, then $L_{1} \cong \operatorname{tr} L_{2} \Rightarrow L_{1} \cong_{\text {en }} L_{2}$, because then en $(L)=\operatorname{Tr}(L) \cap \Sigma$. Clearly " $\simeq_{x}^{x}$ " implies " $\cong x "$. If

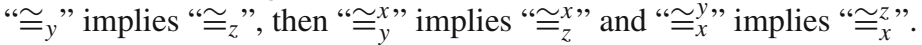

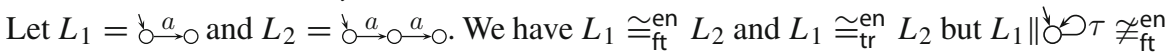

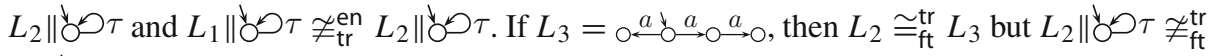
$L_{3} \|^{l} ص \tau$. That is, the grey equivalences in Fig. 1 are not congruences with respect to "\|l". It is also easy (but lengthy) to show with examples that all equivalences in the figure are different. 
Fig. 1 The congruences (black solid) in Theorem 12 and three related non-congruences (grey)

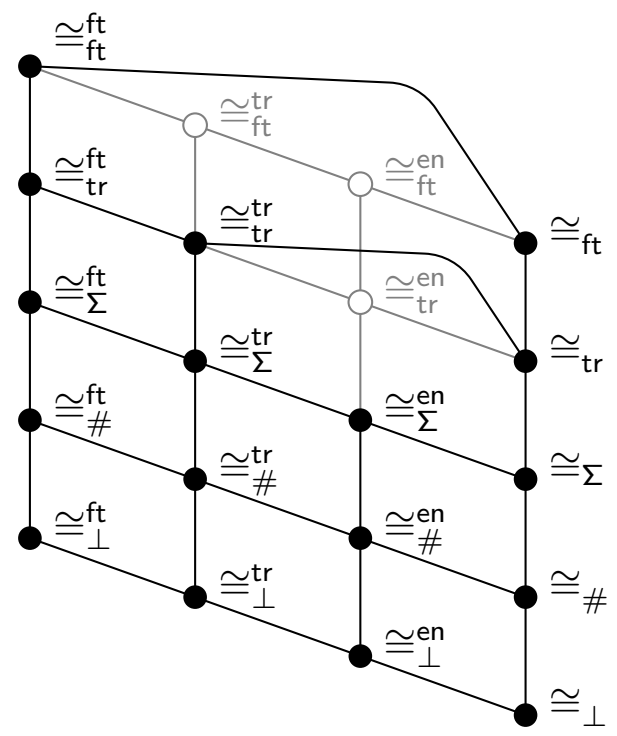

We now investigate the congruence properties of the black equivalences in Fig. 1 with respect to the six operators defined in Sect. 2. The next lemma formulates a principle that is very useful in proving that certain equivalences are congruences.

Lemma 10 Let $f_{1}(L), \ldots, f_{n}(L)$ be functions from LTSs to some sets $D_{1}, \ldots, D_{n}$. Assume

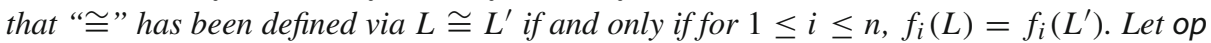
be a unary LTS operator. If, for $1 \leq i \leq n$, there are functions $g_{i}: D_{1} \times \cdots \times D_{n} \rightarrow D_{i}$

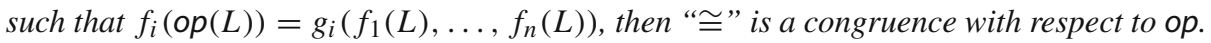

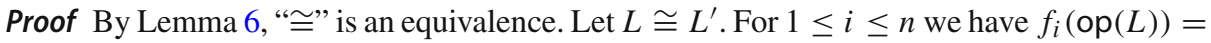
$g_{i}\left(f_{1}(L), \ldots, f_{n}(L)\right)=g_{i}\left(f_{1}\left(L^{\prime}\right), \ldots, f_{n}\left(L^{\prime}\right)\right)=f_{i}\left(\right.$ op $\left.\left(L^{\prime}\right)\right)$, because $f_{j}(L)=f_{j}\left(L^{\prime}\right)$ for $1 \leq j \leq n$. As a consequence, op $(L) \cong \mathrm{op}\left(L^{\prime}\right)$.

For instance, the alphabets of the results of the six operators defined in Sect. 2 were defined via functions on only the alphabets of the argument LTSs. Therefore, " $\cong \Sigma$ " is a congruence by Lemma 10 .

Lemma 11 Let op be any unary LTS operator such that if $L$ is unstable, then also op $(L)$ is unstable. Assume that " $\cong_{y}^{x}$ " and " $\cong_{z}$ " are congruences with respect to op, and that " $\cong_{y}$ " implies " $\cong_{z}$ ". Then " $\simeq_{z}^{x}$ " is a congruence with respect to op.

Proof By Lemma 9, " $\simeq_{z}^{x}$ " is an equivalence. To show that " $\cong_{z}^{x "}$ is a congruence with respect to op, assume that $L_{1} \cong_{z}^{x} L_{2}$.

If $L_{1}$ and $L_{2}$ are both unstable, then by definition $L_{1} \cong_{z} L_{2}$. This implies op $\left(L_{1}\right) \cong_{z}$ op $\left(L_{2}\right)$. Because $L_{1}$ and $L_{2}$ are unstable, also op $\left(L_{1}\right)$ and op $\left(L_{2}\right)$ are unstable. These yield op $\left(L_{1}\right) \cong_{z}^{x}$ op $\left(L_{2}\right)$.

Otherwise $L_{1}$ and $L_{2}$ are both stable. We have $L_{1} \cong_{x} L_{2}, L_{1} \cong_{y}^{x} L_{2}$, and op $\left(L_{1}\right) \cong_{y}^{x}$ op $\left(L_{2}\right)$. If one of op $\left(L_{1}\right)$ and op $\left(L_{2}\right)$ is stable, then also the other one is stable and op $\left(L_{1}\right) \cong_{x}$ op $\left(L_{2}\right)$. These yield op $\left(L_{1}\right) \cong_{z}^{x}$ op $\left(L_{2}\right)$. Otherwise op $\left(L_{1}\right)$ and op $\left(L_{2}\right)$ are unstable and op $\left(L_{1}\right) \cong_{y}$ op $\left(L_{2}\right)$. These yield op $\left(L_{1}\right) \cong_{z}$ op $\left(L_{2}\right)$ and op $\left(L_{1}\right) \cong_{z}^{x}$ op $\left(L_{2}\right)$. 
Table 1 Congruence properties of the black equivalences in Fig. 1

\begin{tabular}{|c|c|c|c|c|c|c|}
\hline & $\|$ & 1 & $\phi$ & $\Phi$ & . & + \\
\hline$\cong_{\mathrm{ft}}^{\mathrm{ft}}$ & $\sqrt{ }$ & $\sqrt{ }$ & $\sqrt{ }$ & $\sqrt{ }$ & $\sqrt{ }$ & $\sqrt{ }$ \\
\hline$\cong_{\mathrm{ft}}$ & $\sqrt{ }$ & $\sqrt{ }$ & $\sqrt{ }$ & $\sqrt{ }$ & $\sqrt{ }$ & \\
\hline$\cong \mathrm{ft}$ & $\sqrt{ }$ & $\sqrt{ }$ & $\sqrt{ }$ & $\sqrt{ }$ & & $\sqrt{ }$ \\
\hline$\simeq_{\mathrm{tr}}^{\mathrm{tr}}$ & $\sqrt{ }$ & $\sqrt{ }$ & $\sqrt{ }$ & $\sqrt{ }$ & $\sqrt{ }$ & $\sqrt{ }$ \\
\hline$\cong_{\mathrm{tr}}$ & $\sqrt{ }$ & $\sqrt{ }$ & $\sqrt{ }$ & $\sqrt{ }$ & $\sqrt{ }$ & $\sqrt{ }$ \\
\hline$\stackrel{\mathrm{ft}}{\Sigma}$ & $\sqrt{ }$ & $\sqrt{ }$ & $\sqrt{ }$ & $\sqrt{ }$ & & $\sqrt{ }$ \\
\hline$\stackrel{\cong}{=} \mathrm{tr}^{\mathrm{r}}$ & $\sqrt{ }$ & $\sqrt{ }$ & $\sqrt{ }$ & $\sqrt{ }$ & & $\sqrt{ }$ \\
\hline$\cong{ }_{\Sigma}^{\mathrm{en}}$ & $\sqrt{ }$ & $\sqrt{ }$ & $\sqrt{ }$ & $\sqrt{ }$ & $\sqrt{ }$ & $\sqrt{ }$ \\
\hline$\cong \Sigma$ & $\sqrt{ }$ & $\sqrt{ }$ & $\sqrt{ }$ & $\sqrt{ }$ & $\sqrt{ }$ & $\sqrt{ }$ \\
\hline$\cong_{\#}^{\mathrm{ft}}$ & $\sqrt{ }$ & $\sqrt{ }$ & $\sqrt{ }$ & & & $\sqrt{ }$ \\
\hline$\cong_{\#}^{\mathrm{tr}}$ & $\sqrt{ }$ & $\sqrt{ }$ & $\sqrt{ }$ & & & $\sqrt{ }$ \\
\hline$\cong_{\#}^{e n}$ & $\sqrt{ }$ & $\sqrt{ }$ & $\sqrt{ }$ & & & $\sqrt{ }$ \\
\hline$\cong_{\#}$ & $\sqrt{ }$ & $\sqrt{ }$ & $\sqrt{ }$ & & $\sqrt{ }$ & $\sqrt{ }$ \\
\hline$\cong_{\perp}^{\mathrm{ft}}$ & $\sqrt{ }$ & $\sqrt{ }$ & $\sqrt{ }$ & $\sqrt{ }$ & & $\sqrt{ }$ \\
\hline$\cong_{\perp}^{\operatorname{tr}}$ & $\sqrt{ }$ & $\sqrt{ }$ & $\sqrt{ }$ & $\sqrt{ }$ & & $\sqrt{ }$ \\
\hline$\cong_{\perp}^{\text {en }}$ & $\sqrt{ }$ & $\sqrt{ }$ & $\sqrt{ }$ & $\sqrt{ }$ & & $\sqrt{ }$ \\
\hline$\cong_{\perp}$ & $\sqrt{ }$ & $\sqrt{ }$ & $\sqrt{ }$ & $\sqrt{ }$ & $\sqrt{ }$ & $\sqrt{ }$ \\
\hline
\end{tabular}

Theorem 12 An equivalence labelling a row in Table 1 is a congruence with respect to the operator labelling a column in the table if and only if the intersection of the row and colum contains $\sqrt{ }$.

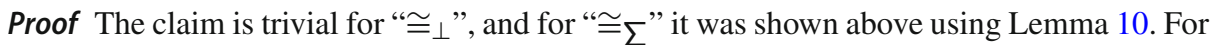

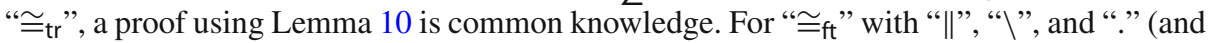
" $\phi ")$, the claim has been shown in [14], and with " $\Phi$ " in [18]. The classic counter-example

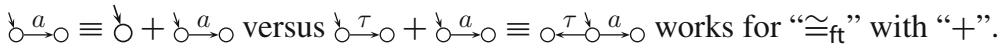

Next we deal with "#". The essence of the proof is that " $\Phi$ " can make the difference between alphabets grow from finite to infinite, while the other five operators cannot (they cannot make the difference grow at all).

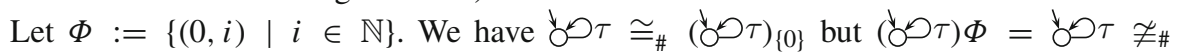

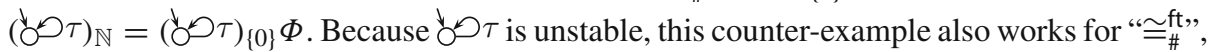

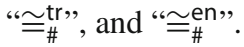

For each of the remaining operators, we provide an injection from $\Sigma\left(\mathrm{op}\left(L_{1}\right)\right) \backslash \Sigma\left(\mathrm{op}\left(L_{2}\right)\right)$ to $\Sigma_{1} \backslash \Sigma_{2}$. This shows that if $\Sigma_{1} \backslash \Sigma_{2}$ is finite, then $\Sigma\left(\mathrm{op}\left(L_{1}\right)\right) \backslash \Sigma\left(\mathrm{op}\left(L_{2}\right)\right)$ is finite as well. The case of $\Sigma\left(\mathrm{op}\left(L_{2}\right)\right) \backslash \Sigma\left(\mathrm{op}\left(L_{1}\right)\right)$ is similar. Because the union of two sets is finite if and only if the sets are finite, the result generalizes to $\Sigma\left(\operatorname{op}\left(L_{1}\right)\right) \# \Sigma\left(\operatorname{op}\left(L_{2}\right)\right)$.

- If $a \in \Sigma\left(L_{1} \| L\right) \backslash \Sigma\left(L_{2} \| L\right)$, then $a \in \Sigma_{1} \backslash \Sigma_{2}$ (and $\left.a \notin \Sigma\right)$.

- If $a \in \Sigma\left(L_{1} \backslash A\right) \backslash \Sigma\left(L_{2} \backslash A\right)$, then $a \in \Sigma_{1} \backslash \Sigma_{2}$ (and $a \notin A$ ).

- If $a \in \Sigma\left(\phi\left(L_{1}\right)\right) \backslash \Sigma\left(\phi\left(L_{2}\right)\right)$, then there is $b \in \Sigma_{1} \backslash \Sigma_{2}$ such that $a=\phi(b)$. Furthermore, each such $a$ has its own $b$, because $\phi\left(b_{1}\right) \neq \phi\left(b_{2}\right)$ implies $b_{1} \neq b_{2}$.

- If $a \in \Sigma\left(L_{1}+L\right) \backslash \Sigma\left(L_{2}+L\right)$, then $a \in \Sigma_{1} \backslash \Sigma_{2}$ (and $\left.a \notin \Sigma\right)$.

- If $a \in \Sigma\left(b . L_{1}\right) \backslash \Sigma\left(b . L_{2}\right)$, then $a \in \Sigma_{1} \backslash \Sigma_{2}$ (and $a \neq b$ ). 
If $L_{1} \cong \mathrm{ft}_{\mathrm{ft}} L_{2}$, then $L_{1} \cong \mathrm{ft} L_{2}$ and $L_{1} \cong \perp L_{2}$. If $L_{1}$ and $L_{2}$ are both stable, then also $L_{1} \Phi$ and $L_{2} \Phi$ are both stable. If $L_{1}$ and $L_{2}$ are both unstable, then also $L_{1} \Phi$ and $L_{2} \Phi$ are both unstable. By the congruence properties of " $\cong_{\mathrm{ft}}$ ", we have $L_{1} \Phi \cong_{\mathrm{ft}} L_{2} \Phi$. Therefore, $L_{1} \Phi \cong \mathrm{ft} L_{2} \Phi$. The remaining claims for " $\cong \mathrm{ft}$ " can be taken from [14] or proven similarly using Lemma 1 . The claims for " $\cong_{\mathrm{tr}}^{\mathrm{tr}}$ " can be proven similarly and are widely known.

The claims for " $\cong$ en" follow from Lemmas 1,10 , and the fact that " $\cong$ en " implies " $\cong \Sigma "$ which is a congruence.

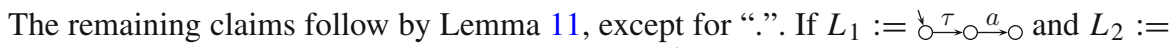

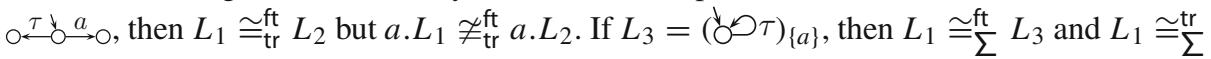
$L_{3}$, but $a \cdot L_{1} ¥_{\Sigma}^{\mathrm{ft}} a \cdot L_{3}$ and $a \cdot L_{1} \varlimsup_{\Sigma}^{\mathrm{tr}} a \cdot L_{3}$. Furthermore, $L_{1} \cong_{y}^{x}{ }^{b} \nabla \tau$ when $x \in\{\mathrm{ft}$, tr, en $\}$ and $y \in\{\#, \perp\}$, but $\Sigma\left(b . L_{1}\right) \neq \Sigma\left(b .{ }^{\downarrow} \supset \tau\right)$ when $b \notin\{a, \tau, \varepsilon\}$, so $b . L_{1} ¥_{y}^{x} b .{ }^{b} \oslash \tau$.

\section{The weakest stability-preserving congruence}

In this section we find the weakest stability-preserving congruence both in the presence and absence of the action prefix operator. This result is central in the study of stability-preserving congruences. It does not assume the congruence property with respect to renaming, so it makes weaker assumptions than the rest of this study.

Theorem 13 The weakest congruence with respect to parallel composition and hiding that

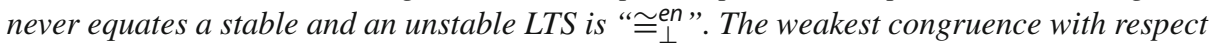
to parallel composition, hiding, and action prefix that never equates a stable and an unstable

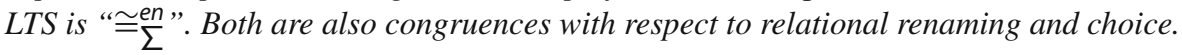

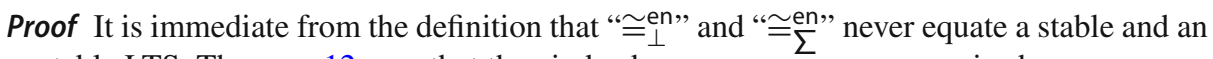
unstable LTS. Theorem 12 says that they indeed are congruences as promised.

It remains to be proven that they are the weakest possible. That is, if a congruence does

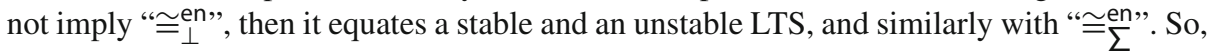
for $x \in\{\perp, \Sigma\}$, we assume that $L_{1} \cong L_{2}$ and $L_{1} \varlimsup_{x}^{\text {en }} L_{2}$, and prove the existence of $L_{1}^{\prime}$ and $L_{2}^{\prime}$ such that one of them is stable, the other is unstable, and $L_{1}^{\prime} \cong L_{2}^{\prime}$.

There are three ways how $L_{1} \nsucceq_{x}^{\text {en }} L_{2}$ may occur.

First, one of $L_{1}$ and $L_{2}$ is stable while the other is unstable. Then they can be used as $L_{1}^{\prime}$ and $L_{2}^{\prime}$.

Second, $L_{1}$ and $L_{2}$ are stable and $L_{1} \nsucceq_{\text {en }} L_{2}$. The latter means that there is $a$ such that $a \in \operatorname{en}\left(L_{1}\right) \backslash$ en $\left(L_{2}\right)$ or $a \in \Sigma_{1} \backslash \Sigma_{2}$ (or the same with the roles of $L_{1}$ and $L_{2}$ swapped).

If $a \in$ en $\left(L_{1}\right) \backslash$ en $\left(L_{2}\right)$, then $L_{2} \backslash\{a\}$ is stable and $L_{1} \backslash\{a\}$ is unstable, so they qualify as $L_{1}^{\prime}$ and $L_{2}^{\prime}$. The same argument applies when $a \in \Sigma_{1} \backslash \Sigma_{2}$ and $a \in \operatorname{en}\left(L_{1}\right)$, because $a \notin \Sigma_{2}$ implies $a \notin \operatorname{en}\left(L_{2}\right)$. The case remains where $a \in \Sigma_{1} \backslash \Sigma_{2}$ and $a \notin \operatorname{en}\left(L_{1}\right)$. Then

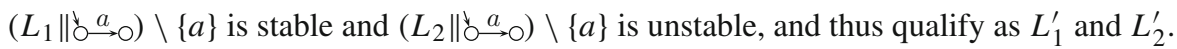

Third, $L_{1}$ and $L_{2}$ are unstable and $L_{1} \oiint_{x} L_{2}$. If $x=\perp$, this is impossible by the definition of " $\cong_{\perp}$ ". So let $x=\Sigma$. There is $a$ such that $a \in \Sigma_{1}$ and $a \notin \Sigma_{2}$ (or the same with the roles of $L_{1}$ and $L_{2}$ swapped). Let $b \notin\{a, \tau, \varepsilon\}$. Then $b . L_{1}$ and $b . L_{2}$ are stable and $a \in \Sigma\left(b . L_{1}\right) \backslash \Sigma\left(b . L_{2}\right)$. So the case has been reduced to an earlier case. 


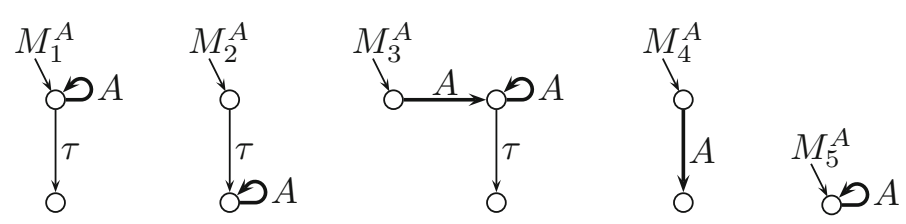

Fig. 2 The LTSs $M_{1}^{A}$ to $M_{5}^{A}$. A thick arc denotes a transition for every element of $A$
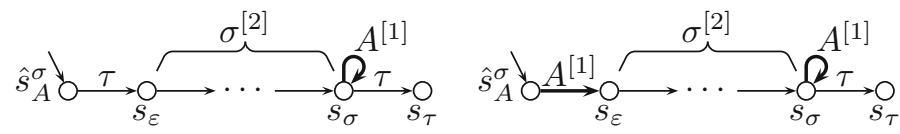

Fig. 3 The two versions of the LTS $L_{A}^{\sigma}$ in the proof of Lemma 14

\section{Proof that Fig. 1 contains all congruences in the region}

In this section we assume that the alphabets of LTSs are finite or countably infinite, and prove that Fig. 1 contains all equivalences that are implied by " $\cong \mathrm{ft}$ ", and are congruences with respect to parallel composition, hiding, and functional renaming. The assumption of countability is only needed for equivalences that do not imply " $\cong$ \#" (and thus not " $\cong \Sigma$ "). The author believes

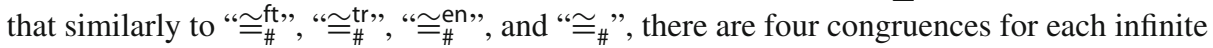
cardinal number in place of "\#”, and, accepting the axiom of choice, that is all. However, the author felt that studying them would have meant going too far from concurrency theory.

For each equivalence " $\approx$ " in Fig. 1, we will prove that any congruence that implies " $\approx$ " but implies neither the nearest equivalence above nor the nearest equivalence above left " $\approx$ " in the figure, is " $\approx$ ". To be able to do so, we first develop ten lemmas. Figure 2 shows five LTSs that are referred to in them. Many of the lemmas use the following assumption:

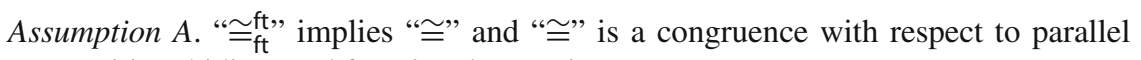
composition, hiding, and functional renaming.

We first prove a lemma that starts with an arbitrary difference between the sets of traces of two equivalent LTSs that have the same alphabet, and, so to speak, amplifies it to the maximal such difference. This result will later be used to prove that if the congruence does not preserve full information on traces, then, both in the case where stability does not matter and in the case where it matters and the LTSs are unstable, it does not preserve any information on traces at all. When stability matters and the LTSs are stable, a similar claim does not hold, because " $\cong \sum$ en" is a congruence. For that case, the lemma presents another result that can be used to show that information on traces beyond the first visible action does not matter.

Lemma 14 Assume $A$. If there are $L_{1}, L_{2}$, and $\sigma$ such that $L_{1} \cong L_{2}, \Sigma_{1}=\Sigma_{2}, \sigma \in \operatorname{Tr}\left(L_{1}\right)$, and $\sigma \notin \operatorname{Tr}\left(L_{2}\right)$, then for every alphabet $A$ we have $\left.M_{1}^{A} \cong(\sigma \tau)\right)_{A}$. If $L_{1}$ and $L_{2}$ are stable, then $M_{3}^{A} \cong M_{4}^{A}$.

Proof Let $\Sigma:=\Sigma_{1}=\Sigma_{2}$, and let $L_{A}^{\sigma}$ be the following LTS (shown in Fig. 3 left):

$$
\begin{aligned}
S_{A}^{\sigma}:= & \left\{s_{\pi} \mid \pi \sqsubseteq \sigma\right\} \cup\left\{\hat{s}_{A}^{\sigma}, s_{\tau}\right\} \quad \text { (all mentioned states are distinct), } \\
\Sigma_{A}^{\sigma}:= & A^{[1]} \cup \Sigma^{[2]}, \text { and } \\
\Delta_{A}^{\sigma}:= & \left\{\left(s_{\pi}, a^{[2]}, s_{\pi a}\right) \mid a \in \Sigma \wedge \pi a \sqsubseteq \sigma\right\} \cup \\
& \left\{\left(s_{\sigma}, a^{[1]}, s_{\sigma}\right) \mid a \in A\right\} \cup
\end{aligned}
$$




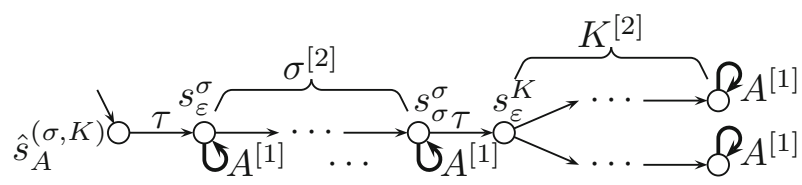

Fig. 4 The LTS $L_{A}^{(\sigma, K)}$ in the proof of Lemma 15

$$
\left\{\left(\hat{s}_{A}^{\sigma}, \tau, s_{\varepsilon}\right),\left(s_{\sigma}, \tau, s_{\tau}\right)\right\}
$$

Let

$$
f\left(L_{i}\right):=\left\lfloor\left(\left\lceil L_{i}\right\rceil^{[2]} \| L_{A}^{\sigma}\right) \backslash \Sigma^{[2]}\right\rfloor[1] .
$$

We have $\Sigma\left(f\left(L_{1}\right)\right)=\Sigma\left(f\left(L_{2}\right)\right)=\Sigma\left(M_{1}^{A}\right)=\Sigma\left((\delta \tau)_{A}\right)=A$. Furthermore, all these four LTSs are unstable.

Because $\sigma \in \operatorname{Tr}\left(L_{1}\right), f\left(L_{1}\right)$ can reach a state of the form $\left(s, s_{\sigma}\right)$. This happens without executing visible actions, because $\Sigma^{[2]}$ is hidden in $f\left(L_{i}\right)$. Then $f\left(L_{1}\right)$ can execute any member of $A$, getting back to $\left(s, s_{\sigma}\right)$. As a consequence, $\operatorname{Tr}\left(f\left(L_{1}\right)\right)=A^{*}$. Because $\left(s_{\sigma}, \tau, s_{\tau}\right) \in \Delta_{A}^{\sigma}$, $f\left(L_{1}\right)$ can continue to $\left(s, s_{\tau}\right)$. Because $s_{\tau}$ has no outgoing transitions, $\operatorname{Tf}\left(f\left(L_{1}\right)\right)=A^{*} \times 2^{A^{+}}$ by Lemma 3. Also $\operatorname{Tf}\left(M_{1}^{A}\right)=A^{*} \times 2^{A^{+}}$by Lemma 3. So $\operatorname{Tf}\left(f\left(L_{1}\right)\right)=\operatorname{Tf}\left(M_{1}^{A}\right)$ and $f\left(L_{1}\right) \stackrel{\mathrm{ft}}{\mathrm{ft}} M_{1}^{A}$.

Because $\sigma \notin \operatorname{Tr}\left(L_{2}\right), f\left(L_{2}\right)$ cannot reach any state of the form $\left(s, s_{\sigma}\right)$, and thus cannot ever execute any member of $A$. We have $\operatorname{Tr}\left(f\left(L_{2}\right)\right)=\{\varepsilon\}$ and $\operatorname{Tf}\left(f\left(L_{2}\right)\right)=\{\varepsilon\} \times 2^{A^{+}}$ $=\operatorname{Tf}\left((\circlearrowleft \tau)_{A}\right)$. So $f\left(L_{2}\right) \cong \mathrm{ft}(\delta \tau \tau)_{A}$.

By the congruence property, $f\left(L_{1}\right) \cong f\left(L_{2}\right)$. We have proven $M_{1}^{A} \cong{ }_{\mathrm{ft}}^{\mathrm{ft}} f\left(L_{1}\right) \cong$

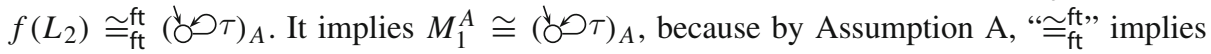

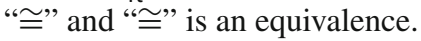

From now on assume that $L_{1}$ and $L_{2}$ are stable. Let $g$ be defined similarly to $f$, except that the transition $\hat{s}_{A}^{\sigma}-\tau \rightarrow s_{\varepsilon}$ is replaced by $\hat{s}_{A}^{\sigma}-a^{[1]} \rightarrow s_{\varepsilon}$ for every $a \in A$ in $L_{A}^{\sigma}$, resulting in the version shown in Fig. 3 right. We have $g\left(L_{1}\right) \cong g\left(L_{2}\right)$ and $\Sigma\left(g\left(L_{1}\right)\right)=\Sigma\left(g\left(L_{2}\right)\right)=$ $\Sigma\left(M_{3}^{A}\right)=\Sigma\left(M_{4}^{A}\right)=A$. Furthermore, all these four LTSs are stable.

For any stable $L, g(L)$ starts by executing an arbitrary member of $A$ and then continues like $f(L)$. As a consequence, $g\left(L_{1}\right) \cong_{\mathrm{ft}}^{\mathrm{ft}} M_{3}^{A}$ and $g\left(L_{2}\right) \cong \cong_{\mathrm{ft}} M_{4}^{A}$, yielding $M_{3}^{A} \cong M_{4}^{A}$.

The next lemma is similar in spirit to the previous one, but this time an arbitrary not alphabetrelated violation against fair testing equivalence is used as the starting point, and the results concern information on the $K$ parts of tree failures.

Lemma 15 Assume $A$. If there are $L_{1}, L_{2}, \sigma$, and $K$ such that $L_{1} \cong L_{2}, \Sigma_{1}=\Sigma_{2},(\sigma, K) \in$ $\operatorname{Tf}\left(L_{1}\right),(\sigma, K) \notin T f\left(L_{2}\right)$, and $\left(\sigma \pi, \pi^{-1} K\right) \notin T f\left(L_{2}\right)$ for every $\pi \sqsubseteq K$, then for every alphabet $A$ we have $M_{1}^{A} \cong M_{2}^{A}$. If $L_{1}$ and $L_{2}$ are stable, then $M_{3}^{A} \cong M_{5}^{A}$.

Proof Let $\Sigma:=\Sigma_{1}=\Sigma_{2}$, and let $L_{A}^{(\sigma, K)}$ be the following LTS (shown in Fig. 4):

$$
\begin{aligned}
S_{A}^{(\sigma, K)}:= & \left\{s_{\pi}^{\sigma} \mid \pi \sqsubseteq \sigma\right\} \cup\left\{s_{\pi}^{K} \mid \varepsilon \neq \pi \sqsubseteq K\right\} \cup\left\{\hat{s}_{A}^{(\sigma, K)}, s_{\varepsilon}^{K}\right\} \\
& \left(\text { all mentioned states are distinct, } s_{\varepsilon}^{K} \text { exists even if } K=\emptyset\right), \\
\Sigma_{A}^{(\sigma, K):=} & A^{[1]} \cup \Sigma^{[2]}, \text { and }
\end{aligned}
$$




$$
\begin{aligned}
\Delta_{A}^{(\sigma, K)}:= & \left\{\left(s_{\pi}^{\sigma}, a^{[2]}, s_{\pi a}^{\sigma}\right) \mid a \in \Sigma \wedge \pi a \sqsubseteq \sigma\right\} \cup \\
& \left\{\left(s_{\pi}^{K}, a^{[2]}, s_{\pi a}^{K}\right) \mid a \in \Sigma \wedge \pi a \sqsubseteq K\right\} \cup \\
& \left\{\left(s_{\pi}^{\sigma}, a^{[1]}, s_{\pi}^{\sigma}\right) \mid a \in A \wedge \pi \sqsubseteq \sigma\right\} \cup \\
& \left\{\left(s_{\pi}^{K}, a^{[1]}, s_{\pi}^{K}\right) \mid a \in A \wedge \pi \in K\right\} \cup \\
& \left\{\left(\hat{s}_{A}^{(\sigma, K)}, \tau, s_{\varepsilon}^{\sigma}\right),\left(s_{\sigma}^{\sigma}, \tau, s_{\varepsilon}^{K}\right)\right\} .
\end{aligned}
$$

Similarly to the previous proof,

$$
f\left(L_{i}\right):=\left\lfloor\left(\left\lceil L_{i}\right\rceil^{[2]} \| L_{A}^{(\sigma, K)}\right) \backslash \Sigma^{[2]}\right\rfloor[1] .
$$

We have $\Sigma\left(f\left(L_{1}\right)\right)=\Sigma\left(f\left(L_{2}\right)\right)=\Sigma\left(M_{1}^{A}\right)=\Sigma\left(M_{2}^{A}\right)=A$. Furthermore, all these four LTSs are unstable.

Let $i \in\{1,2\}$. Trivially $\operatorname{Tr}\left(f\left(L_{i}\right)\right) \subseteq A^{*}$. Without $L_{i}$ moving, $f\left(L_{i}\right)$ can move invisibly from its initial state $\left(\hat{s}_{i}, \hat{s}_{A}^{(\sigma, K)}\right)$ to $\left(\hat{s}_{i}, s_{\varepsilon}^{\sigma}\right)$. Then it can execute any member of $A^{*}$, getting back to $\left(\hat{s}_{i}, s_{\varepsilon}^{\sigma}\right)$ after each transition. Therefore, $\operatorname{Tr}\left(f\left(L_{1}\right)\right)=\operatorname{Tr}\left(f\left(L_{2}\right)\right)=A^{*}$.

Because $(\sigma, K) \in \operatorname{Tf}\left(L_{1}\right), L_{1}$ can execute $\sigma$ and then be in a state $s^{\prime}$ where it cannot execute any element of $K$. So $f\left(L_{1}\right)$ can continue invisibly from $\left(\hat{s}_{1}, s_{\varepsilon}^{\sigma}\right)$ to the state $\left(s^{\prime}, s_{\varepsilon}^{K}\right)$, but cannot continue from there to any state of the form $\left(s, s_{\pi}^{K}\right)$, where $\pi \in K$. That is, $f\left(L_{1}\right)$ can execute any element of $A^{*}$ and then invisibly move to a state from which it cannot continue to a state where it can execute an element of $A$. As a consequence, $\operatorname{Tf}\left(f\left(L_{1}\right)\right)=A^{*} \times 2^{A^{+}}=$ $\operatorname{Tf}\left(M_{1}^{A}\right)$. So $f\left(L_{1}\right) \cong \mathrm{ft}_{\mathrm{ft}} M_{1}^{A}$.

If $f\left(L_{2}\right)$ is in a state of the form $\left(s, s_{\pi}^{\sigma}\right)$, then it can execute any member of $A$ immediately. If $f\left(L_{2}\right)$ is in a state of the form $\left(s, \hat{s}_{A}^{(\sigma, K)}\right)$, then it can execute $\tau$ and enter a state of the previous form. If $f\left(L_{2}\right)$ is in a state of the form $\left(s, s_{\pi}^{K}\right)$ where $\varepsilon \neq \pi \sqsubseteq K$ or $\varepsilon=\pi \sqsubseteq K$, then by $\left(\sigma \pi, \pi^{-1} K\right) \notin \operatorname{Tf}\left(L_{2}\right)$ it can execute invisibly at least one member of $\pi^{-1} K$. That takes it to a state of the form $\left(s^{\prime}, s_{\kappa}^{K}\right)$ where $\kappa \in K$. There it can execute any member of $A$. The case remains where $f\left(L_{2}\right)$ is in a state of the form $\left(s, s_{\varepsilon}^{K}\right)$, where $\varepsilon \nsubseteq K$. Then $L_{2}$ has executed $\sigma$, implying $(\sigma, \emptyset) \in \operatorname{Tf}\left(L_{2}\right)$. On the other hand, $K=\emptyset$ because $\varepsilon \nsubseteq K$. This contradicts $(\sigma, K) \notin \operatorname{Tf}\left(L_{2}\right)$, showing that this case is impossible.

Therefore, $f\left(L_{2}\right)$ cannot reach a state from which it cannot continue to a state where it can execute any member of $A$. We have $\operatorname{Tf}\left(f\left(L_{2}\right)\right)=A^{*} \times\{\emptyset\}$ and $f\left(L_{2}\right) \cong_{\mathrm{ft}}^{\mathrm{ft}} M_{2}^{A}$.

By the congruence property, $f\left(L_{1}\right) \cong f\left(L_{2}\right)$. We have proven $M_{1}^{A} \cong \mathrm{ft} f\left(L_{1}\right) \cong$

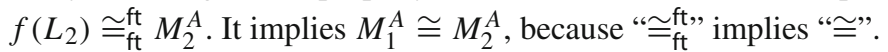

From now on assume that $L_{1}$ and $L_{2}$ are stable. Let $g$ be defined similarly to $f$, except that the transition $\hat{s}_{A}^{(\sigma, K)}-\tau \rightarrow s_{\varepsilon}^{\sigma}$ is replaced by $\hat{s}_{A}^{(\sigma, K)}-a^{[1]} \rightarrow s_{\varepsilon}^{\sigma}$ for every $a \in A$. We have $g\left(L_{1}\right) \cong g\left(L_{2}\right)$ and $\Sigma\left(g\left(L_{1}\right)\right)=\Sigma\left(g\left(L_{2}\right)\right)=\Sigma\left(M_{3}^{A}\right)=\Sigma\left(M_{5}^{A}\right)=A$. Furthermore, all these four LTSs are stable.

For any stable $L, g(L)$ starts by executing an arbitrary member of $A$ and then continues like $f(L)$. As a consequence, $g\left(L_{1}\right) \cong \mathrm{ft}_{\mathrm{ft}} M_{3}^{A}$ and $g\left(L_{2}\right) \cong_{\mathrm{ft}}^{\mathrm{ft}} M_{5}^{A}$, yielding $M_{3}^{A} \cong M_{5}^{A}$.

In the congruences of the form " $\cong x$ " " in Fig. $1, x$ can only be $\mathrm{ft}$, tr, or en. When proving that they suffice, the next two lemmas and Theorem 13 will be used.

Lemma 16 Assume $A$. If there are stable $L_{1}^{\prime}$ and $L_{2}^{\prime}$ such that $L_{1}^{\prime} \cong L_{2}^{\prime}, \Sigma_{1}^{\prime}=\Sigma_{2}^{\prime}$, and $L_{1}^{\prime} ¥_{\mathrm{ft}} L_{2}^{\prime}$, then for any stable $L_{1}$ and $L_{2}$ such that $L_{1} \cong$ tr $L_{2}$ we have $L_{1} \cong L_{2}$.

Proof For any stable $L$, let $f(L):=L \| M_{3}^{\Sigma}$. Clearly $L \equiv L \| M_{5}^{\Sigma}$. By Lemma 15 and the congruence property, $L\left\|M_{5}^{\Sigma} \cong L\right\| M_{3}^{\Sigma}$. So $L \cong f(L)$. Clearly $f(L)$ is stable, $\Sigma(f(L))=$ $\Sigma$, and $\operatorname{Tr}(f(L))=\operatorname{Tr}(L)$. 
By Lemma 4, $(\varepsilon, K) \in \operatorname{Tf}(f(L))$ if and only if $K \cap \operatorname{Tr}(f(L))=\emptyset$. The LTS $M_{3}^{\Sigma}$ may deadlock after any nonempty trace. Therefore, by Lemma 3, if $\sigma \neq \varepsilon$, then $(\sigma, K) \in \operatorname{Tf}(f(L))$ if and only if $\sigma \in \operatorname{Tr}(f(L))$ and $K \subseteq \Sigma(f(L))^{+}$. As a consequence, $\operatorname{Tf}(f(L))$ is determined by $\Sigma(f(L))$ and $\operatorname{Tr}(f(L))$, that is, $\Sigma$ and $\operatorname{Tr}(L)$.

Let $L_{1}$ and $L_{2}$ be stable and $L_{1} \cong_{\operatorname{tr}} L_{2}$. We have $\Sigma_{1}=\Sigma_{2}$ and $\operatorname{Tr}\left(L_{1}\right)=\operatorname{Tr}\left(L_{2}\right)$. These imply $\operatorname{Tf}\left(f\left(L_{1}\right)\right)=\operatorname{Tf}\left(f\left(L_{2}\right)\right)$. Furthermore, $f\left(L_{1}\right)$ and $f\left(L_{2}\right)$ are stable. As a consequence, $f\left(L_{1}\right) \stackrel{\mathrm{ft}}{\mathrm{ft}} f\left(L_{2}\right)$.

Hence $L_{1} \cong f\left(L_{1}\right) \cong \mathrm{ft}_{\mathrm{ft}} f\left(L_{2}\right) \cong L_{2}$, implying $L_{1} \cong L_{2}$.

Lemma 17 Assume $A$. If there are stable $L_{1}^{\prime}$ and $L_{2}^{\prime}$ such that $L_{1}^{\prime} \cong L_{2}^{\prime}, \Sigma_{1}^{\prime}=\Sigma_{2}^{\prime}$, and $L_{1}^{\prime} ¥_{\text {tr }} L_{2}^{\prime}$, then for any stable $L_{1}$ and $L_{2}$ such that $L_{1} \cong$ en $L_{2}$ we have $L_{1} \cong L_{2}$.

Proof For any stable $L$, let $f(L):=L \| M_{4}^{\Sigma}$. Because " $\cong \mathrm{ft}$ " implies " $\cong \mathrm{tr}$ ", the assumptions of Lemma 15 hold. By Lemmas 14 and $15, L \equiv L\left\|M_{5}^{\Sigma} \cong L\right\| M_{3}^{\Sigma} \cong L \| M_{4}^{\Sigma}$. So $L \cong$ $f(L)$. Clearly $f(L)$ is stable and $\Sigma(f(L))=\Sigma$. Because $\operatorname{Tr}\left(M_{4}^{\Sigma}\right)=\Sigma \cup\{\varepsilon\}$, we have $\operatorname{Tr}(f(L))=\operatorname{en}(L) \cup\{\varepsilon\}$. It implies en $(f(L))=\operatorname{en}(L)$.

By Lemma 4, $(\varepsilon, K) \in \operatorname{Tf}(f(L))$ if and only if $K \cap \operatorname{Tr}(f(L))=\emptyset$. By Lemma 3, if $\sigma \neq \varepsilon$, then $(\sigma, K) \in \operatorname{Tf}(f(L))$ if and only if $\sigma \in \operatorname{Tr}(f(L))$ and $K \subseteq \Sigma(f(L))^{+}$. As a consequence, $\operatorname{Tf}(f(L))$ is determined by $\Sigma(f(L))$ and en $(f(L))$, that is, $\Sigma$ and en $(L)$.

Let $L_{1}$ and $L_{2}$ be stable and $L_{1} \cong$ en $L_{2}$. We have $\Sigma_{1}=\Sigma_{2}$ and en $\left(L_{1}\right)=\operatorname{en}\left(L_{2}\right)$. These imply $\operatorname{Tf}\left(f\left(L_{1}\right)\right)=\operatorname{Tf}\left(f\left(L_{2}\right)\right)$. Furthermore, $f\left(L_{1}\right)$ and $f\left(L_{2}\right)$ are stable. As a consequence, $f\left(L_{1}\right) \stackrel{\mathrm{ft}}{\mathrm{ft}} f\left(L_{2}\right)$.

Hence $L_{1} \cong f\left(L_{1}\right) \cong \mathrm{ft} f\left(L_{2}\right) \cong L_{2}$, implying $L_{1} \cong L_{2}$.

In the sequel, we will have to deal with cases where stability does not matter, and with cases where it matters and the LTSs in question are unstable. To exploit results on the latter when dealing with the former, we define a simple operator that, given an LTS, yields an unstable "气 $\mathrm{ft}$--equivalent LTS. We let

$$
\mathrm{us}(L):=L \|^{\dagger} \supset \tau \text {. }
$$

The following lemma tells some properties of us $(L)$.

Lemma 18 Assume A. For every $L$ we have the following.

1. us $(L)$ is unstable.

2. $\operatorname{us}(L) \cong_{f t} L$.

3. If $L$ is unstable, then us $(L) \cong f t h$ and us $(L) \cong L$.

4. If there are $L_{1}$ and $L_{2}$ such that $L_{1} \cong L_{2}, \Sigma_{1}=\Sigma_{2}$, and $L_{1} \oiint_{f t} L_{2}$, then us $(L) \cong$ $L \| M_{1}^{\Sigma}$.

5. If there are $L_{1}$ and $L_{2}$ such that $L_{1} \cong L_{2}, \Sigma_{1}=\Sigma_{2}$, and $L_{1} \nsucceq \operatorname{tr} L_{2}$, then us $(L) \cong$ $(b \tau)_{\Sigma}$.

Proof The first three claims are obvious.

For any $L$, us $(L) \cong$ ft $L \| M_{2}^{\Sigma}$, because they both have $\Sigma$ as the alphabet, they are both unstable, and $M_{2}^{\Sigma}$ never blocks actions of $L$. With the assumptions of the fourth claim, Lemma 15 and the congruence property yield $L\left\|M_{2}^{\Sigma} \cong L\right\| M_{1}^{\Sigma}$. As a consequence, us $(L) \cong$ $L \| M_{1}^{\Sigma}$.

With the assumptions of the last claim, for any $L$, Lemma 14 yields $L\left\|M_{1}^{\Sigma} \cong L\right\|(\mho ص \tau)_{\Sigma}$. Clearly $L \|(\delta \tau)_{\Sigma} \equiv(\delta \tau \tau)_{\Sigma}$, because $(\downarrow \tau)_{\Sigma}$ blocks all visible actions of $L$. Because

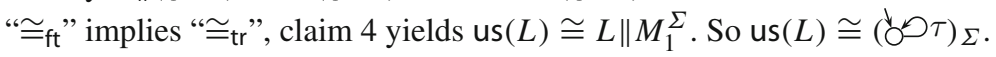


The next lemma tells that if the congruence equates a stable and an unstable LTS, then stability does not matter at all.

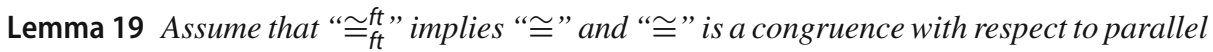
composition and hiding. If there are a stable LTS $L_{s}$ and an unstable LTS $L_{u}$ such that $L_{s} \cong L_{u}$, then

1. $\mathrm{b} \cong \mathrm{v} \tau \tau$, and

2. for any $L, L \cong u s(L)$.

Proof Let $\Sigma:=\Sigma\left(L_{\mathrm{S}}\right) \cup \Sigma\left(L_{\mathrm{u}}\right)$ and $f(L):=\left(L \|_{\mathrm{O}}\right) \backslash \Sigma$. Clearly $f\left(L_{\mathrm{s}}\right) \equiv \mathrm{o}^{\mathrm{o}}$. The alphabet of $f\left(L_{\mathrm{u}}\right)$ is $\emptyset=\Sigma(\emptyset \tau)$, and $\operatorname{Tf}\left(f\left(L_{\mathrm{u}}\right)\right)=\{(\varepsilon, \emptyset)\}=\operatorname{Tf}(\emptyset \smile \tau)$ by Lemma 2(1). Furthermore, $f\left(L_{\mathrm{u}}\right)$ is obviously unstable. So $f\left(L_{\mathrm{u}}\right) \cong_{\mathrm{ft}}^{\mathrm{ft}}{ }^{\downarrow} ص \tau$. These yield $\mathrm{b} \equiv f\left(L_{\mathrm{s}}\right) \cong$ $f\left(L_{\mathrm{u}}\right) \cong \mathrm{ft}$

Let $L$ be any LTS. Clearly $L \equiv L\left\|^{d} \cong L\right\|^{d} \oslash \tau=\mathrm{us}(L)$, so $L \cong \mathrm{us}(L)$.

The next lemma says that if the congruence does not preserve the alphabet, then, in the case of unstable LTSs, it throws away all information on traces and tree failures.

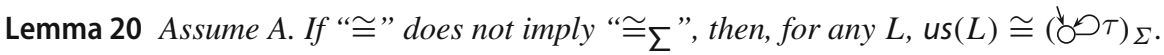

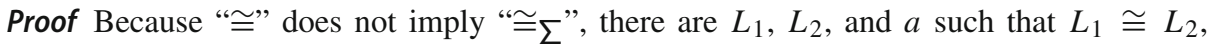
$a \in \Sigma_{1}$, and $a \notin \Sigma_{2}$. Let $\Sigma:=\left(\Sigma_{1} \cup \Sigma_{2}\right) \backslash\{a\}$.

If $L_{1}=a \Rightarrow$, then choose any $b \notin\{a, \tau, \varepsilon\}$ and let $f(L):=\phi\left(L \|_{\{b\}}\right) \backslash \Sigma$, where $\phi(b):=a$ and $\phi(x):=x$ if $x \neq b$. We have $f\left(L_{1}\right)=a \Rightarrow$ but $\neg\left(f\left(L_{2}\right)=a \Rightarrow\right)$. Although $a \notin \Sigma_{2}$, we have $\Sigma\left(f\left(L_{2}\right)\right)=\{a\}$ thanks to ${ }^{\prime}\{b\}$ and $\phi$.

If $\neg\left(L_{1}=a \Rightarrow\right)$, then let $f(L):=\left(L \|_{0} \stackrel{a}{\rightarrow}\right) \backslash \Sigma$. We have $\neg\left(f\left(L_{1}\right)=a \Rightarrow\right)$ but $f\left(L_{2}\right)=a \Rightarrow$.

In both cases, $f\left(L_{1}\right) \cong f\left(L_{2}\right), \Sigma\left(f\left(L_{1}\right)\right)=\Sigma\left(f\left(L_{2}\right)\right)=\{a\}$, and $\operatorname{Tr}\left(f\left(L_{1}\right)\right) \neq$ $\operatorname{Tr}\left(f\left(L_{2}\right)\right)$. By Lemma 18(5), for any $L$, us $(L) \cong\left(\sigma^{\prime} \tau\right)_{\Sigma}$.

If $L_{1} \cong L_{2}$ where $\cong$ is a congruence with respect to parallel composition, then us $\left(L_{1}\right)=$ $L_{1}\left\|\emptyset \tau \cong L_{2}\right\| \emptyset \tau=\operatorname{us}\left(L_{2}\right)$, yielding us $\left(L_{1}\right) \cong \operatorname{us}\left(L_{2}\right)$. Next we prove us $\left(L_{1}\right) \cong$ us $\left(L_{2}\right)$ under five different assumptions, without assuming $L_{1} \cong L_{2}$.

Lemma 21 Assume A. In each of the following situations we have us $\left(L_{1}\right) \cong u s\left(L_{2}\right)$.

1. If $L_{1} \cong{ }_{f t} L_{2}$.

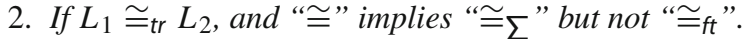

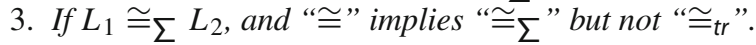

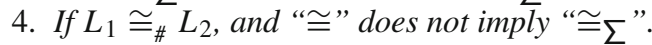

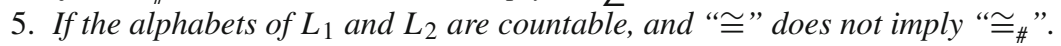

Proof 1. By Lemma $18(2)$, us $\left(L_{1}\right) \cong_{\mathrm{ft}} L_{1} \cong_{\mathrm{ft}} L_{2} \cong_{\mathrm{ft}} \mathrm{us}\left(L_{2}\right)$. So us $\left(L_{1}\right) \cong_{\mathrm{ft}} \mathrm{us}\left(L_{2}\right)$. This implies us $\left(L_{1}\right) \cong{ }_{\mathrm{ft}}^{\mathrm{ft}}$ us $\left(L_{2}\right)$, because us $\left(L_{1}\right)$ and us $\left(L_{2}\right)$ are unstable by Lemma $18(1)$. By assumption A, this implies us $\left(L_{1}\right) \cong$ us $\left(L_{2}\right)$.

2. Let $L \in\left\{L_{1}, L_{2}\right\}$ and $f(L):=L \| M_{1}^{\Sigma}$. It is unstable because of $M_{1}^{\Sigma}$, so $f\left(L_{1}\right) \cong{ }_{\perp}^{\perp} f\left(L_{2}\right)$. We have $\Sigma(f(L))=\Sigma$. Because $M_{1}^{\Sigma}$ may deadlock after any trace, Lemma 3 yields $\operatorname{Tf}(f(L))=\left\{(\sigma, K) \mid \sigma \in \operatorname{Tr}(L) \wedge K \subseteq \Sigma^{+}\right\}$. Because $L_{1} \cong_{\operatorname{tr}} L_{2}$, we have $\Sigma_{1}=\Sigma_{2}$ and $\operatorname{Tr}\left(L_{1}\right)=\operatorname{Tr}\left(L_{2}\right)$. These yield $f\left(L_{1}\right) \cong{ }_{\mathrm{ft}}^{\mathrm{ft}} f\left(L_{2}\right)$, implying $f\left(L_{1}\right) \cong f\left(L_{2}\right)$. The part 
of the condition after "and" justifies the use of Lemma 18(4), implying us $\left(L_{1}\right) \cong f\left(L_{1}\right) \cong$ $f\left(L_{2}\right) \cong \mathrm{us}\left(L_{2}\right)$.

3. The condition $L_{1} \cong \Sigma L_{2}$ means that $\Sigma_{1}=\Sigma_{2}$. By Lemma 18(5), us $\left(L_{1}\right) \cong(\oslash \tau)_{\Sigma_{1}}=$ $(\emptyset \tau)_{\Sigma_{2}} \cong \mathrm{us}\left(L_{2}\right)$.

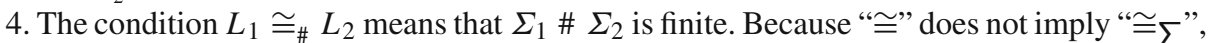
there are $L_{1}^{\prime}, L_{2}^{\prime}$, and $a$ such that $L_{1}^{\prime} \cong L_{2}^{\prime}, a \in \Sigma_{1}^{\prime}$, and $a \notin \Sigma_{2}^{\prime}$. Let $\Sigma:=\left(\Sigma_{1}^{\prime} \cup \Sigma_{2}^{\prime}\right) \backslash\{a\}$. By Lemma 20 , ${ }^{\dagger} ص \tau \cong \operatorname{us}\left(L_{2}^{\prime} \backslash \Sigma\right) \cong \operatorname{us}\left(L_{1}^{\prime} \backslash \Sigma\right) \cong\left({ }^{\prime} ص \tau\right)_{\{a\}}$, where us $\left(L_{2}^{\prime} \backslash \Sigma\right) \cong$ us $\left(L_{1}^{\prime} \backslash \Sigma\right)$ follows from $L_{1}^{\prime} \cong L_{2}^{\prime}$. Therefore, ${ }^{\prime} ص \tau \cong(\delta \nabla \tau)\{a\}$.

Choose any $b$ such that $\tau \neq b \neq \varepsilon$. Let $\phi(a):=b$ and $\phi(x):=x$ when $x \neq a$. We have

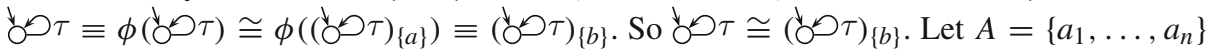
be any finite alphabet. For $0 \leq i<n$, we have $(\emptyset \tau)_{\left\{a_{1}, \ldots, a_{i}\right\}} \equiv(\emptyset \tau)_{\left\{a_{1}, \ldots, a_{i}\right\}} \| \emptyset \tau$

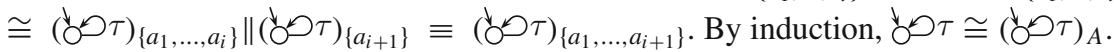

If $\Sigma_{1} \# \Sigma_{2}$ is finite, then also $\Sigma_{1} \backslash \Sigma_{2}$ and $\Sigma_{2} \backslash \Sigma_{1}$ are finite. By Lemma 20, us $\left(L_{1}\right) \cong$

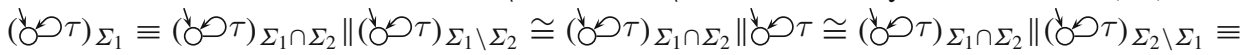
$(\circlearrowleft \tau)_{\Sigma_{2}} \cong \mathrm{us}\left(L_{2}\right)$.

5. By the assumption, there are $L_{1}^{\prime}$ and $L_{2}^{\prime}$ such that $L_{1}^{\prime} \cong L_{2}^{\prime}$ and $\Sigma_{1}^{\prime} \backslash \Sigma_{2}^{\prime}$ is infinite. By

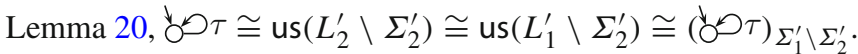

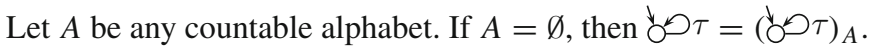

Otherwise there is $a \in A$. Because every infinite set contains a countably infinite subset, there is a bijection $f$ from $A$ to a subset of $\Sigma_{1}^{\prime} \backslash \Sigma_{2}^{\prime}$. A surjection $\phi$ from $\Sigma_{1}^{\prime} \backslash \Sigma_{2}^{\prime}$ to $A$ is obtained by letting $\phi(x):=b$ if $x=f(b)$ and $\phi(x):=a$ if there is no $b$ such that $x=f(b)$.

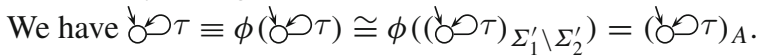

So both $A=\emptyset$ and $A \neq \emptyset$ yield $\emptyset^{\downarrow} ص \tau \cong(\emptyset \tau)_{A}$. We conclude us $\left(L_{1}\right) \cong(\emptyset \tau)_{\Sigma_{1}} \cong$

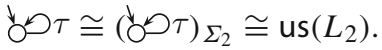

We now have sufficient machinery to prove the main result. We deal first with the case where stability matters.

Lemma 22 Let $x$ be any of $\mathrm{ft}$, tr, and en, and let $\operatorname{prev}(x)$ be the previous one (if $x \neq \mathrm{ft}$ ). Let $y$ be any of $\mathrm{ft}, \mathrm{tr}, \Sigma$, \#, and $\perp$, and let prev(y) be the previous one (if $y \neq \mathrm{ft}$ ). Assume

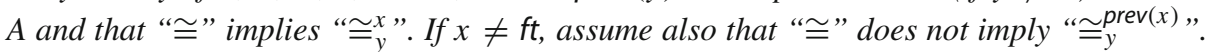

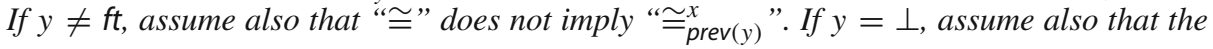

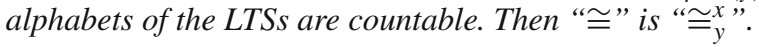

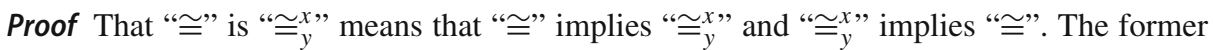
was given in the assumption part of the lemma. Our task is to prove the latter for each $x$ and $y$. So we assume that $L_{1}$ and $L_{2}$ are arbitrary LTSs such that $L_{1} \cong_{y}^{x} L_{2}$, and we have to prove that $L_{1} \cong L_{2}$.

The definition of " $\cong x$ " implies that $L_{1}$ and $L_{2}$ are both stable or both unstable. If $L_{1}$ and $L_{2}$ are stable, then $L_{1} \cong_{x} L_{2}$. There are three cases.

- If $x=\mathrm{ft}$, then $L_{1}$ and $L_{2}$ are stable and $L_{1} \cong_{\mathrm{ft}} L_{2}$. By definition, $L_{1} \cong_{\mathrm{ft}}^{\mathrm{ft}} L_{2}$. It implies $L_{1} \cong L_{2}$ by assumption A.

- If $x=\operatorname{tr}$, then $L_{1} \cong \operatorname{tr} L_{2}$ and there are $L_{1}^{\prime}$ and $L_{2}^{\prime}$ such that $L_{1}^{\prime} \cong L_{2}^{\prime}$ but $L_{1}^{\prime} ¥_{y}^{\mathrm{ft}} L_{2}^{\prime}$. Because $L_{1}^{\prime} \cong L_{2}^{\prime}$ implies $L_{1}^{\prime} \cong{ }_{y}^{\operatorname{tr}} L_{2}^{\prime}$, this means that $L_{1}^{\prime}$ and $L_{2}^{\prime}$ are both stable, $L_{1}^{\prime} \varlimsup_{\mathrm{ft}} L_{2}^{\prime}$, and $\Sigma_{1}^{\prime}=\Sigma_{2}^{\prime}$. Lemma 16 yields $L_{1} \cong L_{2}$. 
- If $x=$ en, then $L_{1} \cong$ en $L_{2}$ and there are $L_{1}^{\prime}$ and $L_{2}^{\prime}$ such that $L_{1}^{\prime} \cong L_{2}^{\prime}$ but $L_{1}^{\prime} \dddot{y}_{y}^{\text {tr }} L_{2}^{\prime}$. Because $L_{1}^{\prime} \cong L_{2}^{\prime}$ implies $L_{1}^{\prime} \cong{ }_{y}^{e n} L_{2}^{\prime}$, this means that $L_{1}^{\prime}$ and $L_{2}^{\prime}$ are both stable, $L_{1}^{\prime} ¥_{\text {tr }} L_{2}^{\prime}$, and $\Sigma_{1}^{\prime}=\Sigma_{2}^{\prime}$. Lemma 17 yields $L_{1} \cong L_{2}$.

If $L_{1}$ and $L_{2}$ are unstable, then Lemma 18(3) yields $L_{1} \cong \mathrm{us}\left(L_{1}\right)$ and us $\left(L_{2}\right) \cong L_{2}$. We will soon show that the assumptions of Lemma 21 hold. By it, us $\left(L_{1}\right) \cong$ us $\left(L_{2}\right)$, yielding $L_{1} \cong L_{2}$.

Because $L_{1}$ and $L_{2}$ are unstable, $L_{1} \cong_{y}^{x} L_{2}$ implies $L_{1} \cong{ }_{y}$. This gives the first condition of Lemma 21(1) to (4). The first condition of (5) is in the assumptions of the

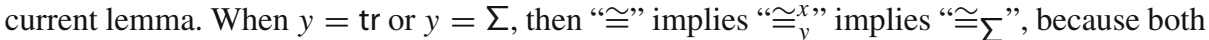

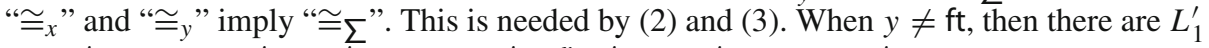
and $L_{2}^{\prime}$ such that $L_{1}^{\prime} \cong L_{2}^{\prime}$ yielding $L_{1}^{\prime} \cong{ }_{y}^{x} L_{2}^{\prime}$, but $L_{1}^{\prime} \varlimsup_{\text {prev }(y)}^{x} L_{2}^{\prime}$. They are unstable and

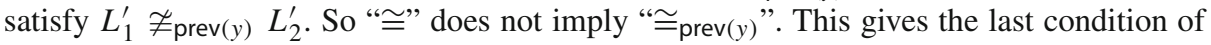
(2) to (5) and completes the checking of the assumptions of Lemma 21.

Before continuing, it is perhaps a good idea to discuss a bit the fact that Lemma 22 refers to three equivalences that are grey in Fig. 1. First, in some cases " $\simeq_{\operatorname{prev}(y)}^{x}$ " or " $\cong{ }_{y}^{\operatorname{prev}(x)}$ " is grey. This is not a problem, because the lemma does not assume that it is a congruence. It only assumes that there are $L_{1}^{\prime}$ and $L_{2}^{\prime}$ such that $L_{1}^{\prime} \cong L_{2}^{\prime}$ but $L_{1}^{\prime} ¥_{\operatorname{prev}(y)}^{x} L_{2}^{\prime}$ and $L_{1}^{\prime} ¥_{y}^{\operatorname{prev}(x)} L_{2}^{\prime}$.

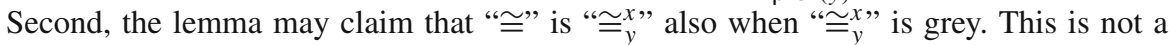
problem, because the lemma does not promise but assumes that "으" is a congruence. The lemma says that if there is a congruence with the assumed properties, then it is " $\cong x$ ". If " $\cong{ }_{y}^{x}$ ", is not a congruence, then, with the chosen $x$ and $y$, no congruences satisfy the assumptions of the lemma.

The case remains where stability does not matter.

Lemma 23 Let $y$ be any of $f t, t r, \Sigma$, \#, and $\perp$, and let $\operatorname{prev}(y)$ be the previous one (if $y \neq f t$ ).

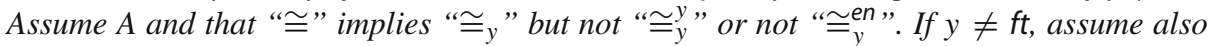

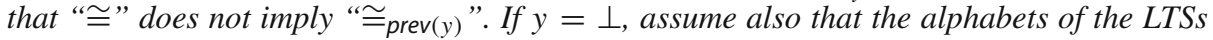

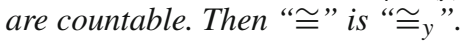

Proof We first show that there are $L_{1}^{\prime \prime}$ and $L_{2}^{\prime \prime}$ such that one of them is stable, the other is unstable, and $L_{1}^{\prime \prime} \cong L_{2}^{\prime \prime}$. By the assumptions, there are $L_{1}^{\prime}$ and $L_{2}^{\prime}$ such that $L_{1}^{\prime} \cong L_{2}^{\prime}$ and

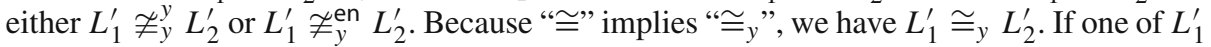
and $L_{2}^{\prime}$ is stable and the other is unstable, then they qualify as $L_{1}^{\prime \prime}$ and $L_{2}^{\prime \prime}$. Because $L_{1}^{\prime} \cong_{y} L_{2}^{\prime}$, the only remaining possibility is that $L_{1}^{\prime}$ and $L_{2}^{\prime}$ are both stable and $L_{1}^{\prime} ¥_{\text {en }} L_{2}^{\prime}$. The latter

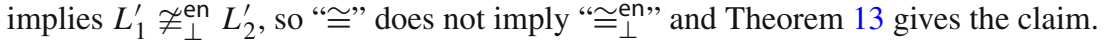

As a consequence, for every $L$, Lemma $19(2)$ yields $L \cong$ us $(L)$. If $L_{1} \cong y L_{2}$, then $L_{1} \cong \mathrm{us}\left(L_{1}\right) \cong \mathrm{us}\left(L_{2}\right) \cong L_{2}$ by Lemma 21 and the fact that " $\cong$ tr" implies " $\cong \Sigma$ ". Therefore,

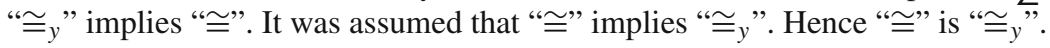

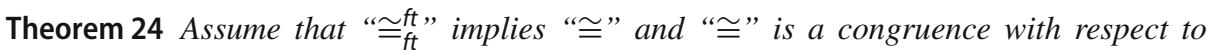

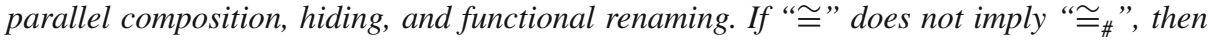

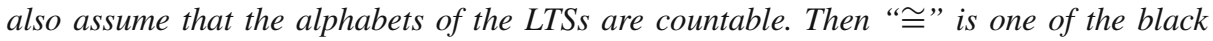
equivalences in Fig. 1.

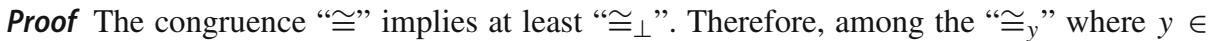
$\{\mathrm{ft}, \mathrm{tr}, \Sigma, \#, \perp\}$ that it implies, there is a first one. For this $y$, if " $\cong$ " does not imply the next

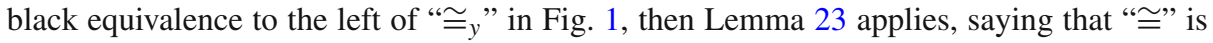
“@y". 


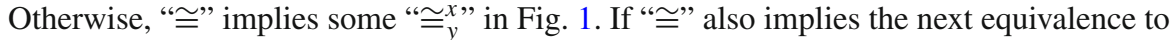
the left (if $x \neq \mathrm{ft}$ ) or the next equivalence above (if $y \neq \mathrm{ft}$ ), go there even if it is grey. Repeat

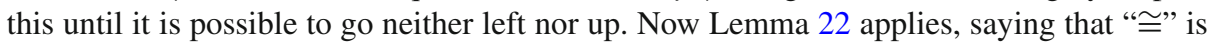

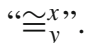

As a consequence, the 20 equivalences in Fig. 1 contain all congruences that are implied by " $\cong \mathrm{ft}$ " (making the countability assumption where needed). In Sect. 3 we proved that the black ones among them are congruences and the grey ones are not.

\section{A somewhat general theory on adding stability preservation}

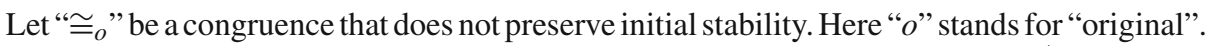
The goal is to find all congruences that are implied by " $\cong_{o}^{o}$ " (that is, " $\cong_{o}$ " $\cap$ " $\cong \perp$ "), in terms of the congruences that are implied by " $\cong_{o}$ ". (There is no point in studying the case where

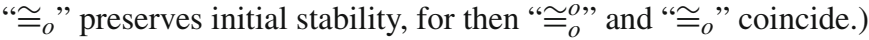

The first part of our work only needs very weak assumptions:

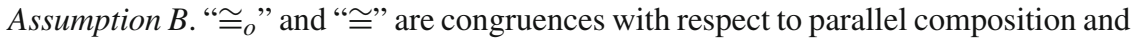

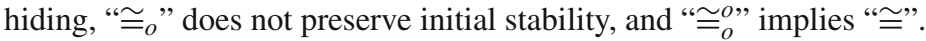

The following simple lemma will be used often.

Lemma 25 Assume B. If $L_{1} \cong{ }_{o} L_{2}$ and $L_{1} \cong \perp L_{2}$, then $L_{1} \cong L_{2}$.

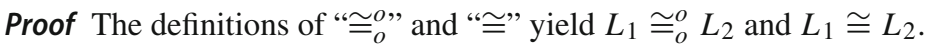

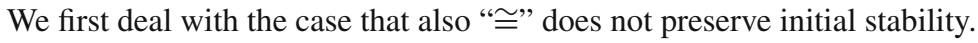

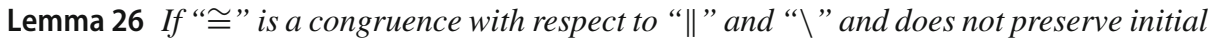
stability, then there is an unstable LTS $U$ such that ${ }^{\circ} \mathrm{O} \cong U$.

Proof By the assumption, there are a stable LTS $L_{\mathrm{s}}$ and an unstable LTS $L_{\mathrm{u}}$ such that $L_{\mathrm{s}} \cong L_{\mathrm{u}}$.

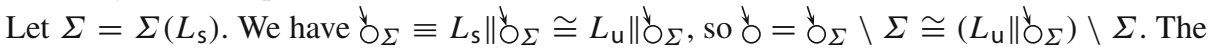
latter is unstable.

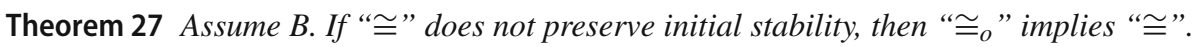

Proof Assume that $L_{1} \cong{ }_{o} L_{2}$. We have to show $L_{1} \cong L_{2}$.

Let $U$ be like in Lemma 26. Because " $\cong$ " " is a congruence, we have $L_{1}\left\|U \cong{ }_{o} L_{2}\right\| U$. Both $L_{1} \| U$ and $L_{2} \| U$ are unstable because $U$ is unstable. Therefore, Lemma 25 yields $L_{1}\left\|U \cong L_{2}\right\| U$.

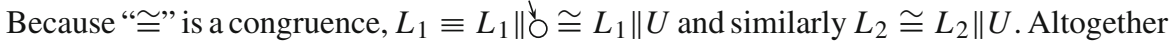
$L_{1} \cong L_{1}\left\|U \cong L_{2}\right\| U \cong L_{2}$ giving $L_{1} \cong L_{2}$.

In the rest of this section " $\cong$ " does preserve initial stability. Therefore, " $\cong$ " can be represented in the form " $\cong x$ ", where " $\cong$ " ' is a binary relation such that for stable LTSs $L_{1} \cong{ }_{x} L_{2} \Leftrightarrow$ $L_{1} \cong L_{2}$, and " $\cong$ " ' is a binary relation such that for unstable LTSs $L_{1} \cong{ }_{y} L_{2} \Leftrightarrow L_{1} \cong L_{2}$.

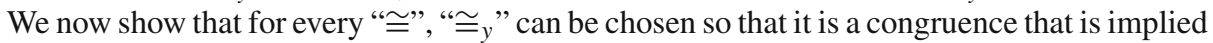

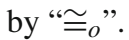

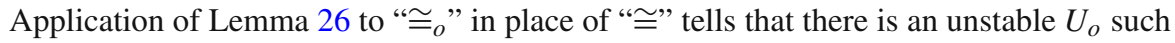
that ${ }^{\circ} \cong_{o} U_{o}$. We define $L_{1} \cong_{\tau} L_{2}: \Leftrightarrow L_{1}\left\|U_{o} \cong L_{2}\right\| U_{o}$, and prove that " $\cong_{\tau}$ " qualifies as "œy". 
Lemma 28 Assume $B$. Then “ $\simeq_{\tau}$ ” is a congruence.

Proof Because " $\cong$ " is an equivalence, Lemma 6 implies that " $\cong_{\tau}$ " is an equivalence as well.

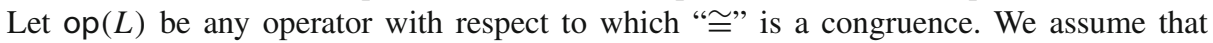
$L_{1} \cong_{\tau} L_{2}$ and show that $\operatorname{op}\left(L_{1}\right) \cong_{\tau} \operatorname{op}\left(L_{2}\right)$.

By the definition, $L_{1}\left\|U_{o} \cong L_{2}\right\| U_{o}$. Because " $\cong$ " is a congruence, we have op $\left(L_{1} \| U_{o}\right) \cong$ op $\left(L_{2} \| U_{o}\right)$ and op $\left(L_{1} \| U_{o}\right)\left\|U_{o} \cong \operatorname{op}\left(L_{2} \| U_{o}\right)\right\| U_{o}$.

Let $L \in\left\{L_{1}, L_{2}\right\}$. Because ${ }^{\circ} \cong_{o} U_{o}$ and " $\cong_{o}$ " is a congruence, we have $L \equiv L \|^{b} \cong_{o}$ $L \| U_{o}$ and $\operatorname{op}(L)\left\|U_{o} \cong{ }_{o} \operatorname{op}\left(L \| U_{o}\right)\right\| U_{o}$. Both op $(L) \| U_{o}$ and $\operatorname{op}\left(L \| U_{o}\right) \| U_{o}$ are unstable, so Lemma 25 yields op $(L) \| U_{o} \cong$ op $\left(L \| U_{o}\right) \| U_{o}$.

We have op $\left(L_{1}\right)\left\|U_{o} \cong \mathrm{op}\left(L_{1} \| U_{o}\right)\right\| U_{o} \cong \mathrm{op}\left(L_{2} \| U_{o}\right)\left\|U_{o} \cong \mathrm{op}\left(L_{2}\right)\right\| U_{o}$. Therefore, $\mathrm{op}\left(L_{1}\right) \cong_{\tau} \mathrm{op}\left(L_{2}\right)$.

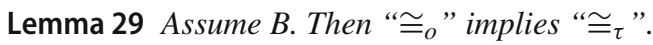

Proof Assume that $L_{1} \cong{ }_{o} L_{2}$. Because " $\cong$ " is a congruence, we have $L_{1}\left\|U_{o} \cong{ }_{o} L_{2}\right\| U_{o}$. Since $L_{1} \| U_{o}$ and $L_{2} \| U_{o}$ are unstable, Lemma 25 yields $L_{1}\left\|U_{o} \cong L_{2}\right\| U_{o}$, that is, $L_{1} \cong_{\tau} L_{2}$.

Lemma 30 Assume $B$ and that $L_{1}$ and $L_{2}$ are unstable. Then $L_{1} \cong L_{2}$ if and only if $L_{1} \cong{ }_{\tau} L_{2}$.

Proof Assume that $L_{1} \cong L_{2}$. Because " $\cong$ " is a congruence, we have $L_{1}\left\|U_{o} \cong L_{2}\right\| U_{o}$, that is, $L_{1} \cong_{\tau} L_{2}$.

Assume that $L_{1} \cong L_{2}$. That is, $L_{1}\left\|U_{o} \cong L_{2}\right\| U_{o}$. Like above, we have $L_{1} \equiv L_{1} \|$ o $\cong_{o} L_{1} \| U_{o}$. Because $L_{1}$ and $L_{1} \| U_{o}$ are unstable, Lemma 25 yields $L_{1} \cong L_{1} \| U_{o}$. Similar reasoning yields $L_{2} \cong L_{2} \| U_{o}$. Altogether $L_{1} \cong L_{1}\left\|U_{o} \cong L_{2}\right\| U_{o} \cong L_{2}$.

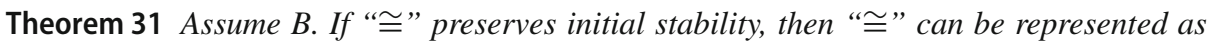

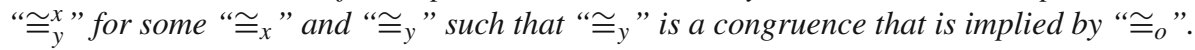

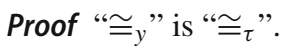

The fact that " $\cong$ en " is a congruence tells that a corresponding theorem for " $\cong$ " must be more complicated. This is because the congruence " $\cong \sum_{\Sigma}^{e n}$ " is implied by " $\simeq_{\operatorname{tr}}^{\operatorname{tr}}$ ", but no congruence

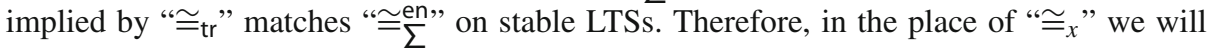
use a relation that checks that $L_{1} \cong{ }_{\text {en }} L_{2}$ and, roughly speaking, for each $a \in \operatorname{en}\left(L_{1}\right)$, the behaviours of $L_{1}$ after $a$ and $L_{2}$ after $a$ are in a congruence that is implied by " $\cong_{0}$ ". Our proof relies on much stronger assumptions than assumption $\mathrm{B}$. The first part of the assumptions is shown below, and the second part will be presented after we have developed the necessary notions.

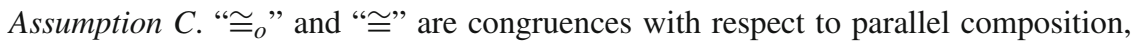

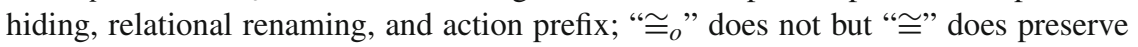

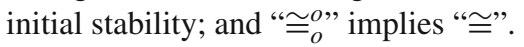

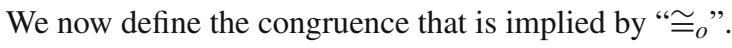

Definition 32 For any LTSs $L_{1}$ and $L_{2}$, we define $L_{1} \cong L_{2}$ if and only if there is $x \notin$ $\Sigma_{1} \cup \Sigma_{2} \cup\{\tau, \varepsilon\}$ such that $x . L_{1} \cong x . L_{2}$.

Lemma 33 Assume $C$. If $L_{1} \cong L_{2}$, then $a . L_{1} \cong a . L_{2}$ holds for all $a \notin\{\tau, \varepsilon\}$. 


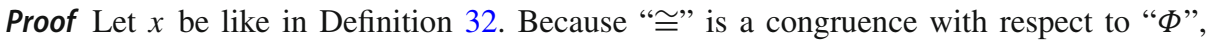
$x . L_{1} \cong x . L_{2}$ implies $\left(x . L_{1}\right)\{(x, a)\} \cong\left(x . L_{2}\right)\{(x, a)\}$. Because $x \notin \Sigma_{1} \cup \Sigma_{2}$ we have $a \cdot L_{1}=\left(x \cdot L_{1}\right)\{(x, a)\} \cong\left(x . L_{2}\right)\{(x, a)\}=a \cdot L_{2}$, yielding $a \cdot L_{1} \cong a \cdot L_{2}$.

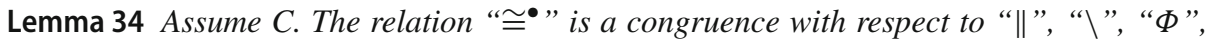
and " $a$. ."

Proof Let $L_{1}, L_{2}$ and $L_{3}$ be LTSs, $a$ a visible action, $A$ a set of visible actions, and $\Phi$ a set of pairs of visible actions. Let $x$ be a visible action that is not in $A \cup\{a\} \cup \Sigma_{1} \cup \Sigma_{2} \cup \Sigma_{3}$ and not in any pair of $\Phi$. By Lemma 33, whenever $L \cong{ }^{\prime} L^{\prime}$ holds below for some $L$ and $L^{\prime}$, we have $x . L \cong x . L^{\prime}$.

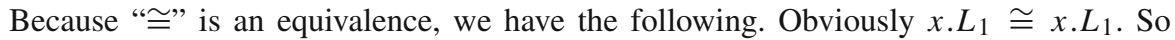

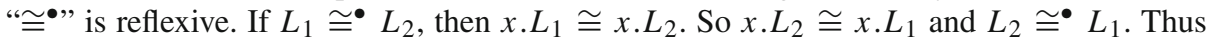

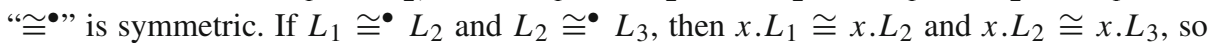

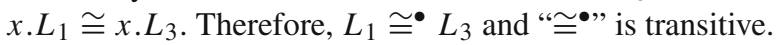

Because $x \notin \Sigma_{1} \cup \Sigma_{2} \cup \Sigma_{3} \cup\{\tau, \varepsilon\}$, we have $x .\left(L_{i} \| L_{3}\right) \equiv\left(x . L_{i}\right) \|\left(x . L_{3}\right)$ when $i=1$ or

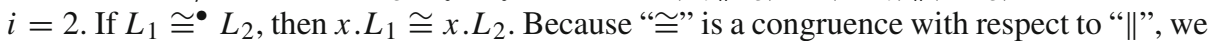
have $\left(x . L_{1}\right)\left\|\left(x . L_{3}\right) \cong\left(x . L_{2}\right)\right\|\left(x . L_{3}\right)$. So $x .\left(L_{1} \| L_{3}\right) \cong x .\left(L_{2} \| L_{3}\right)$ and $L_{1}\left\|L_{3} \cong L_{2}\right\| L_{3}$. Similar reasoning proves $L_{3}\left\|L_{1} \cong L_{3}\right\| L_{2}$. Therefore, " $\cong$ " ' is a congruence with respect to " $\|$ ".

Because $x \notin A$, we have $x .\left(L_{i} \backslash A\right) \equiv\left(x . L_{i}\right) \backslash A$ when $i=1$ or $i=2$. If $L_{1} \cong L_{2}$,

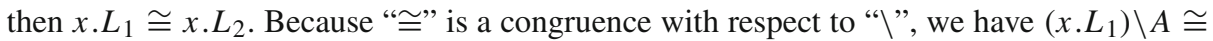
$\left(x . L_{2}\right) \backslash A$. So $x .\left(L_{1} \backslash A\right) \cong x .\left(L_{2} \backslash A\right)$ and $L_{1} \backslash A \cong L_{2} \backslash A$. Therefore, " $\cong \bullet "$ is a congruence with respect to " $\backslash$ ".

By the choice of $x$, we have $x \cdot\left(L_{i} \Phi\right) \equiv\left(x . L_{i}\right) \Phi$ when $i=1$ or $i=2$. If $L_{1} \cong L_{2}$,

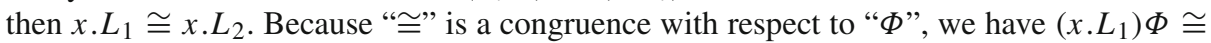
$\left(x . L_{2}\right) \Phi$. So $x .\left(L_{1} \Phi\right) \cong x .\left(L_{2} \Phi\right)$ and $L_{1} \Phi \cong L_{2} \Phi$. Therefore, " $\cong \bullet "$ is a congruence with respect to " $\Phi$ ".

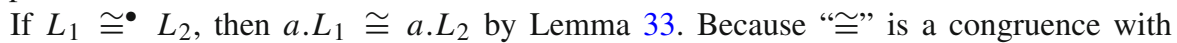

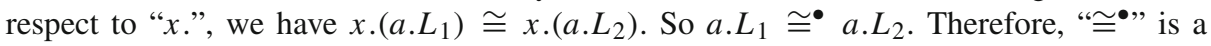
congruence with respect to " $a$.". By choosing $a$ so that it is not in $\Sigma_{1} \cup \Sigma_{2}$ we also get $\tau . L_{1}=\left(a . L_{1}\right) \backslash\{a\} \cong\left(a . L_{2}\right) \backslash\{a\}=\tau . L_{2}$, because we have already shown that " $\cong \bullet "$ is a congruence with respect to " $\backslash$ ". Thus " $\cong \bullet "$ is a congruence with respect to " $\tau$.".

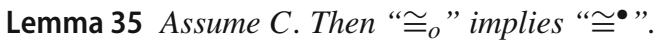

Proof Assume that $L_{1} \cong_{o} L_{2}$. Let $x \notin \Sigma_{1} \cup \Sigma_{2} \cup\{\tau, \varepsilon\}$. By the congruence property, we have $x . L_{1} \cong{ }_{0} x . L_{2}$. Since $x . L_{1}$ and $x . L_{2}$ are stable, Lemma 25 yields $x . L_{1} \cong x . L_{2}$. That is, $L_{1} \cong L_{2}$.

We will soon make it precise what we mean by the behaviour of a stable LTS after a visible action. As a preparatory step, let $\Sigma$ be a set of visible actions and $x$ a visible action. We define $\underset{\mathrm{H}}{x} \underset{\mathrm{x}}{\longrightarrow} \mathrm{S}$ as the two-state LTS whose alphabet is $\Sigma \cup\{x\}$ and transitions are $\left\{\left(\hat{s}_{x}, x, s_{x}\right)\right\} \cup$ $\left\{\left(s_{x}, a, s_{x}\right) \mid a \in \Sigma\right\}$.

Let $L=(S, \Sigma, \Delta, \hat{s})$ be a stable LTS, $a \in \Sigma$, and $x \notin \Sigma \cup\{\tau, \varepsilon\}$. We will soon use the LTS $L_{x}^{a}=L\{(a, x)(a, a)\} \|\left.\stackrel{x}{\longrightarrow}\right|^{\infty} \Sigma$. To get intuition for it, we now show that it is isomorphic to the reachable part of $L^{\prime}=\left(S^{\prime}, \Sigma^{\prime}, \Delta^{\prime}, \hat{s}^{\prime}\right)$, where $\hat{s}^{\prime}$ is a new state (that is, $\left.\hat{s}^{\prime} \notin S\right), S^{\prime}=S \cup\left\{\hat{s}^{\prime}\right\}, \Sigma^{\prime}=\Sigma \cup\{x\}$, and $\Delta^{\prime}=\Delta \cup\left\{\left(\hat{s}^{\prime}, x, s\right) \mid(\hat{s}, a, s) \in \Delta\right\}$ (Fig. 5).

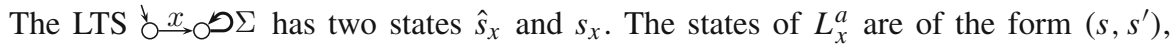
where $s \in S$ and $s^{\prime} \in\left\{\hat{s}_{x}, s_{x}\right\}$. Because the alphabet of both $L\{(a, x)(a, a)\}$ and $\stackrel{x}{\longrightarrow} \underset{\square}{\longrightarrow}$ is 

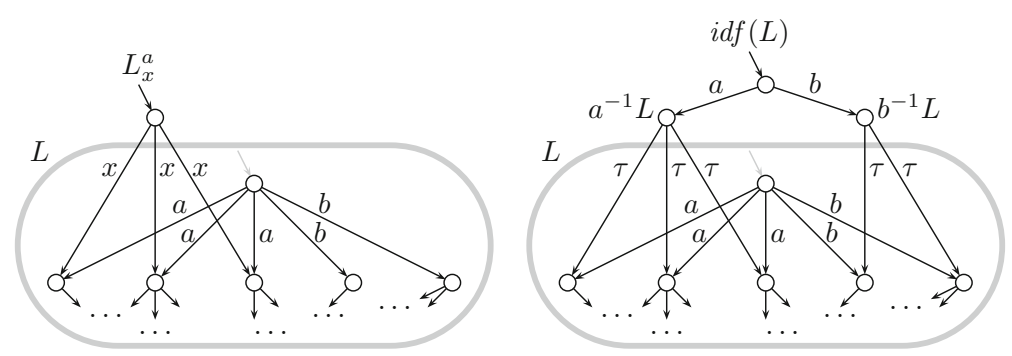

Fig. 5 Illustrating $L_{x}^{a}$ (left), $a^{-1} L$, and $i d f(L)$ (right)

$\Sigma \cup\{x\}$, and because $\stackrel{x}{\longrightarrow} \underset{\sim}{\longrightarrow}$ has no $\tau$-transitions, the transitions of $L_{x}^{a}$ are of three forms: $\left(s, \hat{s}_{x}\right)-x \rightarrow\left(s^{\prime}, s_{x}\right)$ where (thanks to the renaming) $\left(s, a, s^{\prime}\right) \in \Delta ;\left(s, s_{x}\right)-b \rightarrow\left(s^{\prime}, s_{x}\right)$ where $b \in \Sigma$ and $\left(s, b, s^{\prime}\right) \in \Delta$; and $\left(s, s_{x}^{\prime}\right)-\tau \rightarrow\left(s^{\prime}, s_{x}^{\prime}\right)$ where $\left(s, \tau, s^{\prime}\right) \in \Delta$ and $s_{x}^{\prime} \in\left\{\hat{s}_{x}, s_{x}\right\}$. Once $\hat{s}_{x}$ has been left, it cannot be re-entered. Furthermore, $L$ is stable. Therefore, the states of the form $\left(s, \hat{s}_{x}\right)$ where $s \neq \hat{s}$ are unreachable. The states of the form $\left(s, s_{x}\right)$ and their outgoing transitions constitute a copy of the reachable part of $L$, in addition to which there is the transition $\left(\hat{s}, \hat{s}_{x}\right)-x \rightarrow\left(s, s_{x}\right)$ for every $(\hat{s}, a, s)$ of the reachable part of $L$.

The LTS $L_{x}^{a} \backslash\{x\}$ is otherwise similar, but it lacks $x$ in its alphabet and its initial transitions are labelled with $\tau$ instead of $x$. It is independent of the choice of $x$ (as long as $x \notin \Sigma \cup\{\tau, \varepsilon\}$ ). In structural operational semantics,

$$
\frac{L-a \rightarrow L^{\prime}}{L_{x}^{a} \backslash\{x\}-\tau \rightarrow L^{\prime}}
$$

and that is all $L_{x}^{a} \backslash\{x\}$ can do. From now on we denote it with $a^{-1} L$. That is, if $L$ is a stable LTS, $a \in \Sigma$, and $x \notin \Sigma \cup\{\tau, \varepsilon\}$, then we define

$$
a^{-1} L=\left(L\{(a, x)(a, a)\} \| \stackrel{x}{\longrightarrow} \mathrm{O}^{\Sigma} \Sigma\right) \backslash\{x\} .
$$

It is easy to check that $\Sigma\left(a^{-1} L\right)=\Sigma$.

Lemma 36 Assume $C$. If $L_{1} \cong L_{2}$ where $L_{1}$ and $L_{2}$ are stable, then $\Sigma_{1}=\Sigma_{2}$, en $\left(L_{1}\right)=$ en $\left(L_{2}\right)$, and $a^{-1} L_{1} \cong a^{-1} L_{2}$ for every $a \in$ en $\left(L_{1}\right)$.

Proof Theorem 13 yields $L_{1} \cong{ }_{\text {en }} L_{2}$, that is, $\Sigma_{1}=\Sigma_{2}$ and en $\left(L_{1}\right)=\operatorname{en}\left(L_{2}\right)$. The congruence properties of " $\cong$ " and the definition of $a^{-1} L$ yield $x \cdot\left(a^{-1} L_{1}\right) \cong x \cdot\left(a^{-1} L_{2}\right)$, from

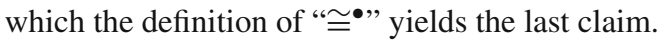

To prove the converse of Lemma 36, we discuss the construction of $L$, when $a^{-1} L$ is given for each $a \in \operatorname{en}(L)$. Then we present the assumptions we will use in addition to assumption $\mathrm{C}$.

Let $A$ be a set of visible actions and $L_{a}$ be an LTS for each $a \in A$. If $A$ is finite, then it is of the form $\left\{a_{1}, \ldots, a_{n}\right\}$, where the $a_{i}$ are distinct from each other. We define finite deterministic choice between $a_{1} \cdot L_{a_{1}}, \ldots, a_{n} \cdot L_{a_{n}}$ as $\sum_{a \in A} a \cdot L_{a}=a_{1} \cdot L_{a_{1}}+\cdots+a_{n} \cdot L_{a_{n}}$. Infinite deterministic choice is the natural extension to infinite $A$, and deterministic choice is finite or infinite deterministic choice. "Deterministic" signifies that $\sum_{a \in A} a . L_{a}$ has precisely one initial transition for each $a \in A$, and no other initial transitions.

Definition 37 If $L$ is stable, then by its initially deterministic form we mean

$$
i d f(L)=\sum_{a \in \operatorname{en}(L)} a \cdot\left(a^{-1} L\right) .
$$




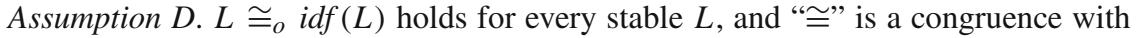
respect to infinite deterministic choice.

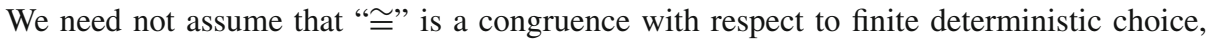
because Lemma 40 will tell that it is. However, we first focus on the big picture, and present the result where Assumption D is needed.

Lemma 38 Assume $C$ and $D$. If $L_{1}$ and $L_{2}$ are stable, en $\left(L_{1}\right)=$ en $\left(L_{2}\right)$, and $a^{-1} L_{1} \cong$ $a^{-1} L_{2}$ for every $a \in$ en $\left(L_{1}\right)$, then $L_{1} \cong L_{2}$.

Proof Clearly $\operatorname{idf}(L)$ is stable, so Assumption D and Lemma 25 imply $L_{1} \cong \operatorname{idf}\left(L_{1}\right)$ and $\operatorname{idf}\left(L_{2}\right) \cong L_{2}$. Because $a^{-1} L_{1} \cong a^{-1} L_{2}$ and $a$ is visible, by Lemma $33, a .\left(a^{-1} L_{1}\right)$ $\cong a \cdot\left(a^{-1} L_{2}\right)$ for each $a \in \operatorname{en}\left(L_{1}\right)=\operatorname{en}\left(L_{2}\right)$. Thus Lemma 40 (in the finite case) and Assumption D (in the infinite case) yield $i d f\left(L_{1}\right) \cong i d f\left(L_{2}\right)$.

We can now prove a result that resembles Lemma 30 and can be used to characterize the

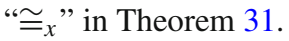

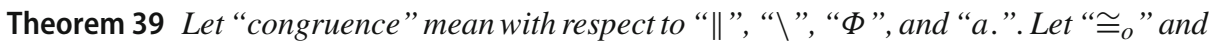

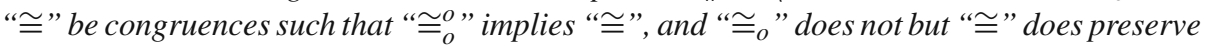

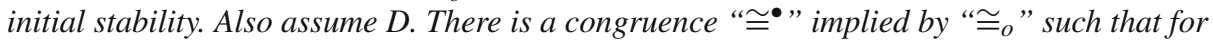
stable LTSs, $L_{1} \cong L_{2}$ if and only if $\Sigma_{1}=\Sigma_{2}$, en $\left(L_{1}\right)=$ en $\left(L_{2}\right)$, and $a^{-1} L_{1} \cong a^{-1} L_{2}$ for every $a \in \operatorname{en}\left(L_{1}\right)$.

Proof The assumptions in the theorem imply Assumption C. By Lemmas 34 and 35, the relation in Definition 32 is a congruence implied by “ $\cong_{o}$ ". Lemmas 36 and 38 give the last claim.

The use of Assumption D reduces the generality of this theorem. The rest of this section is devoted to a brief analysis on conditions where Assumption D holds. Based on it, we will see in the next section that the first half of Assumption D is not a problem with CFFD equivalence.

Next we show that if we restrict ourselves to LTSs $L$ such that en $(L)$ is finite whenever $L$ is stable, then the latter part of D need not be assumed. We do that by showing that the choice operator can be constructed from parallel composition and functional renaming, if the LTSs are stable. If $A$ and $B$ are sets of visible actions, we define $C(A, B):=A C \stackrel{A}{\longleftarrow} \stackrel{B}{\longrightarrow} D B$, where each thick arrow denotes a transition for each member of the label of the arrow.

Lemma 40 If $L_{1}$ and $L_{2}$ are stable, then

$$
L_{1}+L_{2} \equiv\left\lfloor\left\lceil L_{1}\right\rceil^{[1]}\left\|\left\lceil L_{2}\right\rceil^{[2]}\right\| C\left(\Sigma_{1}^{[1]}, \Sigma_{2}^{[2]}\right)\right\rfloor_{[1,2]} .
$$

Proof Let the right hand side be called $R$. Because of the renaming, the alphabets of $\left\lceil L_{1}\right\rceil^{[1]}$ and $\left\lceil L_{2}\right\rceil^{[2]}$ are disjoint and the alphabet of $C(\ldots)$ is their union. So all visible transitions of $R$ are either joint transitions by $\left\lceil L_{1}\right\rceil^{[1]}$ and $C(\ldots)$ or joint transitions by $\left\lceil L_{2}\right\rceil^{[2]}$ and $C(\ldots)$. Thanks to $\lfloor\ldots\rfloor_{[1,2]}$, they have the labels that are used in $L_{1}$ and $L_{2}$. Because $C(\ldots)$ has no $\tau$-transitions, all $\tau$-transitions of $R$ arise from $\tau$-transitions of $L_{1}$ or $\tau$-transitions of $L_{2}$.

Let the states of $C(\ldots)$ be called $c_{1}, \hat{c}$, and $c_{2}$. The initial state of $R$ is $\left(\hat{s}_{1}, \hat{s}_{2}, \hat{c}\right)$. It has no $\tau$-transitions, because $L_{1}$ and $L_{2}$ are stable. It has the transitions $\left(\hat{s}_{1}, \hat{s}_{2}, \hat{c}\right)-a \rightarrow\left(s_{1}, \hat{s}_{2}, c_{1}\right)$ where $\hat{s}_{1}-a \rightarrow s_{1}$ is a transition of $L_{1}$, and $\left(\hat{s}_{1}, \hat{s}_{2}, \hat{c}\right)-a \rightarrow\left(\hat{s}_{1}, s_{2}, c_{2}\right)$ where $\hat{s}_{2}-a \rightarrow s_{2}$ is a transition of $L_{2}$. When in $c_{1}, C(\ldots)$ stays there forever, blocks $L_{2}$ in $\hat{s}_{2}$, and lets $L_{1}$ proceed freely. Therefore, states of the form $\left(s_{1}, \hat{s}_{2}, c_{1}\right)$ and their outgoing transitions constitute a copy of $L_{1}$. A similar claim holds about $\left(\hat{s}_{1}, s_{2}, c_{2}\right)$ and $L_{2}$. 
This construction does not generalize to infinite choice, because infinite parallel composition is a problematic thing. For instance, if $L={ }_{0}^{b} \stackrel{a}{\rightarrow}$, then $a \in \operatorname{Tr}(L\|L\| \cdots)$, but if $L={ }^{b} \stackrel{\tau}{\rightarrow} \mathrm{O} \stackrel{a}{\rightarrow} 0$, then $a \notin \operatorname{Tr}(L\|L\| \cdots)$, because an infinite number of $\tau$-transitions would be needed to enable $a$. This example warns that we cannot take extensions of the congruence property to infinite

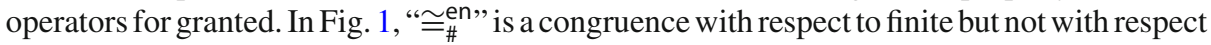
to infinite (nondeterministic!) choice, because of the counter-example where $L_{i}={ }^{\downarrow} \tau \rightarrow 0\{2 i\}$ and $L_{i}^{\prime}=\stackrel{b}{\rightarrow} \mathrm{O}\{2 i, 2 i+1\}$ for $i \in \mathbb{N}$. The author has found neither a proof nor a counter-example to $\operatorname{idf}\left(L_{1}\right) \cong i d f\left(L_{2}\right)$ when $L_{1}$ and $L_{2}$ are stable, $\Sigma_{1}=\Sigma_{2}$, en $\left(L_{1}\right)=\operatorname{en}\left(L_{2}\right)$ is infinite, $a^{-1} L_{1} \cong a^{-1} L_{2}$ for each $a \in \operatorname{en}\left(L_{1}\right), C$ is assumed, and $D$ is not.

A relation that satisfies assumption $C$ (and, by Lemma 40, is thus a congruence with respect to finite choice) but is not a congruence with respect to deterministic infinite choice, would be an oddity. So the inability of our theory to deal with such relations without an extra assumption is perhaps not a big drawback. The first part of Assumption D is, however, significant. It says that if $L$ is stable, then $L \cong_{o} i d f(L)$. For instance, Milner's observation equivalence does not satisfy it.

The only difference between a stable $L$ and $i d f(L)$ is that the choice between initial transitions with the same label is postponed to a choice between $\tau$-transitions after the initial transition (see Fig. 5). This is formalized next.

Lemma 41 If $L$ is a stable LTS, then idf $(L) \equiv\left(S^{\prime}, \Sigma, \Delta^{\prime}, \hat{s}^{\prime}\right)$, where $S^{\prime}=S \cup\left\{\hat{s}^{\prime}\right\} \cup$ $\left\{s_{a} \mid a \in\right.$ en $\left.(L)\right\}$, the added states are distinct from each other and the states in $S$, and $\Delta^{\prime}$ is obtained from $\Delta$ by adding, for each $a \in e n(L)$, the transition $\hat{s}^{\prime}-a \rightarrow s_{a}$ and for each $(\hat{s}, a, s) \in \Delta$ the transitions $s_{a}-\tau \rightarrow s$.

Proof For each $a \in \operatorname{en}(L), a^{-1} L$ and $a .\left(a^{-1} L\right)$ have the same alphabet as $L$, and thus also $i d f(L)$ has the same alphabet. The LTS characterization $L_{x}^{a} \backslash\{x\}$ of $a^{-1} L$ picks the part of $L$ that starts with $a$-transitions, and hides the initial $a$-transitions. The construction of $i d f(L)$ adds an $a$-transition to the front of $a^{-1} L$ and puts the resulting $a .\left(a^{-1} L\right)$ together.

\section{Application to CFFD equivalence}

In this section we apply the theory in the previous section to prove that the stability-preserving CFFD equivalence implies precisely 79 congruences. Throughout this section the word "congruence" means congruence with respect to parallel composition, hiding, relational renaming, action prefix, and infinite deterministic choice. To keep this section reasonably short, we skip some proofs that consist of routine checking, and also skip the definitions that are only needed in such proofs. The definitions can be found in [17].

The state $s_{0}$ diverges, denoted with $s_{0}-\tau^{\omega} \rightarrow$, if and only if there are states $s_{i}$ for every $i>0$ such that $s_{0}-\tau \rightarrow s_{1}-\tau \rightarrow \ldots$. The set of divergence traces of $L$ is $\operatorname{Div}(L)=$ $\left\{\sigma \in \Sigma^{*} \mid \exists s: \hat{s}=\sigma \Rightarrow s-\tau^{\omega} \rightarrow\right\}$. The notation $s=\sigma \Rightarrow$ extends naturally to infinite sequences of visible actions. The set of infinite traces of $L$ is $\operatorname{lnf}(L)=\left\{\xi \in \Sigma^{\omega} \mid \hat{s}=\xi \Rightarrow\right\}$. If $\hat{s}-a_{1} \rightarrow s_{1}-a_{2} \rightarrow \ldots$ is an infinite path of $L$, then the projection of $a_{1} a_{2} \cdots$ on visible actions is either a divergence trace (if it is finite) or an infinite trace (if it is infinite).

The set of stable failures of $L$ is $\operatorname{Sf}(L)=\left\{(\sigma, A) \in \Sigma^{*} \times 2^{\Sigma} \mid \exists s: \hat{s}=\sigma \Rightarrow s \wedge\right.$ $\forall a \in A \cup\{\tau\}: \neg(s-a \rightarrow)\}$. That is, a stable failure is a pair consisting of a trace and a set of visible actions such that $L$ can execute the trace and then be in a stable state where it cannot execute any element of the set. Assume that $\hat{s}=\sigma \Rightarrow s$. If a stable state can be reached from 
Fig. 6 All congruences with respect to a. $L, L \backslash A, L \Phi$, and $L \| L^{\prime}$ that are implied by CFFD equivalence. There is a path from

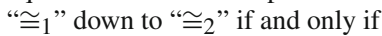

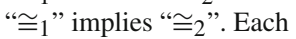
congruence preserves all sets listed along the paths from it

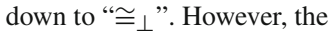
definition of a congruence need not mention those sets that can be determined from the sets that are mentioned [17]

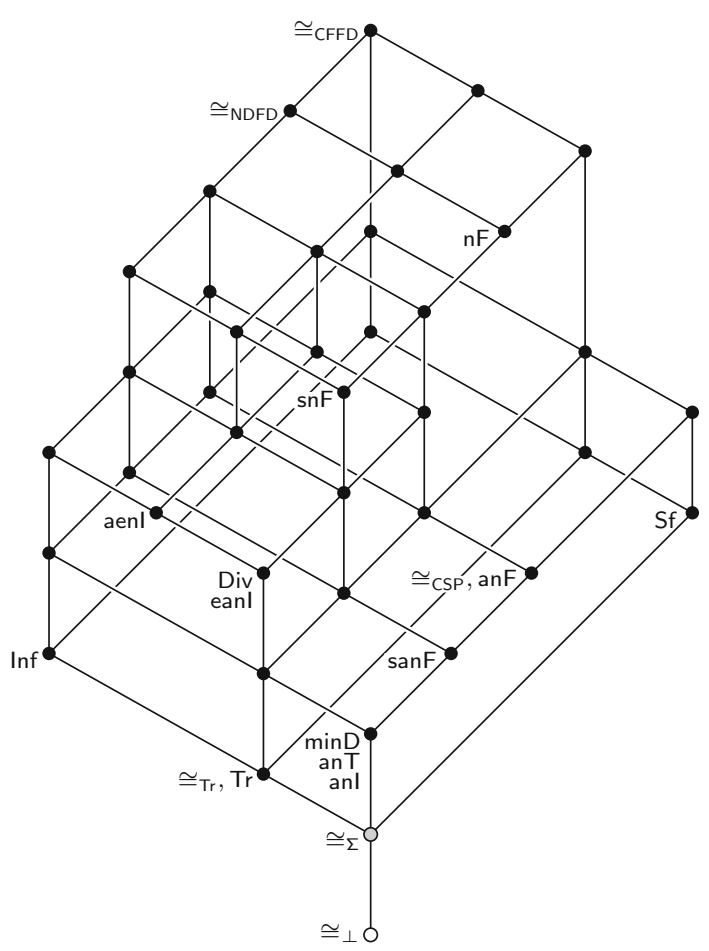

$s$ via $\tau$-transitions, then $(\sigma, \emptyset)$ is a stable failure of $L$, and otherwise $\sigma$ is a divergence trace of $L$. Therefore, $\operatorname{Tr}(L)=\operatorname{Div}(L) \cup\{\sigma \mid(\sigma, \emptyset) \in \operatorname{Sf}(L)\}$.

The LTSs $L_{1}$ and $L_{2}$ are $C F F D$-equivalent, that is, $L_{1} \cong_{\text {CFFD }} L_{2}$, if and only if $\Sigma_{1}=\Sigma_{2}$, $\operatorname{Sf}\left(L_{1}\right)=\operatorname{Sf}\left(L_{2}\right), \operatorname{Div}\left(L_{1}\right)=\operatorname{Div}\left(L_{2}\right)$, and $\operatorname{lnf}\left(L_{1}\right)=\operatorname{lnf}\left(L_{2}\right)$.

It is obvious from the definition and Lemma 41 that if $L$ is a stable LTS, then $L \cong$ CFFD $\operatorname{idf}(L)$. That is, the first part of Assumption D holds for CFFD equivalence. It is also clear that $L \cong$ CFFD $\tau$. $L$ for any LTS $L$.

CFFD equivalence implies precisely 40 congruences with respect to parallel composition, hiding, relational renaming and action prefix. They are shown in Fig. 6 [17]. The figure

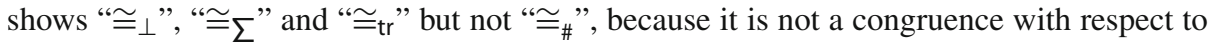
relational renaming. The figure also shows the CSP failures divergences equivalence [15]. For convenience, we call other congruences in the figure than " $\cong_{\perp}$ " and " $\cong \Sigma$ " black, and "气 $\cong$ " is grey.

The congruences use two kinds of traces, two kinds of divergence traces, four kinds of infinite traces, and five kinds of failures. For instance, $\operatorname{anT}(L)$ and $\operatorname{anl}(L)$ are the traces and infinite traces whose prefixes are not divergence traces; $\operatorname{minD}(L)$ is the divergence traces whose proper prefixes are in $\operatorname{anT}(L) ; \operatorname{anF}(L)$ is the stable failures whose trace part is in $\operatorname{anT}(L)$; and $\operatorname{sanF}(L)$ is the same with the additional requirement that if $(\sigma,\{a\}) \in \operatorname{sanF}(L)$, then $\sigma a \notin \operatorname{Div}(L)$. The CSP failures divergences equivalence results from requiring that $\Sigma_{1}=\Sigma_{2}, \operatorname{anF}\left(L_{1}\right)=\operatorname{anF}\left(L_{2}\right), \operatorname{minD}\left(L_{1}\right)=\operatorname{minD}\left(L_{2}\right)$, and $\operatorname{anl}\left(L_{1}\right)=\operatorname{anl}\left(L_{2}\right)$. When this holds, then also $\operatorname{anT}\left(L_{1}\right)=\operatorname{anT}\left(L_{2}\right)$ and $\operatorname{sanF}\left(L_{1}\right)=\operatorname{sanF}\left(L_{2}\right)$. The set anl is needed here although it is typically not used with CSP, beacuse there something is assumed to the effect that the LTSs are finitely branching, which we do not assume. 
Lemma 42 If $L$ is stable, then en $(L)=\operatorname{Tr}(L) \cap \Sigma=\{a \in \Sigma \mid(\varepsilon,\{a\}) \notin S f(L)\}=$ $(\min D(L) \cup \operatorname{an} T(L)) \cap \Sigma$.

Proof Because $\neg(\hat{s}-\tau \rightarrow)$, we have $\operatorname{Tr}(L) \cap \Sigma=\{a \in \Sigma \mid \hat{s}-a \rightarrow\}=\operatorname{en}(L)$. For the same reason, only $\hat{s}$ can introduce stable failures of the form $(\varepsilon, A)$. Furthermore, $\varepsilon \notin \operatorname{minD}(L)$ because $\neg(\hat{s}-\tau \rightarrow)$. Therefore, each $a \in \operatorname{Tr}(L) \cap \Sigma$ is either a minimal divergence trace or an always nondivergent trace.

We mention without proof that these 40 congruences are also congruences with respect to choice between stable LTSs, and thus with respect to infinite deterministic choice. Intuitively, this is because $\operatorname{Tr}\left(\sum L_{i}\right)=\bigcup \operatorname{Tr}\left(L_{i}\right)$; similarly with Div and Inf; minD has a similar formula where only the minimal elements of the union are kept; anT, anl, eanl, and aenl have similar formulas with (minimal) divergence traces used to fix the result; the failures of any kind of the form $(\varepsilon, A)$ are dealt with similarly to Lemma 42; and the failures $(\sigma, A)$ with $\sigma \neq \varepsilon$ have somewhat similar formulas as anT.

Furthermore, if " $\cong_{x}$ " is any of the black congruences, then " $\cong_{x}^{x}$ " is a congruence, because by Lemma 42 it is the intersection of " $\cong_{x}$ " and " $\cong \sum_{\Sigma}^{e n " . ~ B e c a u s e ~ i t ~ p r e s e r v e s ~ i n i t i a l ~ s t a b i l i t y, ~ i t ~}$ is possible to reason the stable failures of the form $(\varepsilon, A)$ of the result of the choice between any LTSs from the stable failures of the component LTSs. Thus " $\cong_{x}^{x}$ " is a congruence also with respect to both finite and infinite choice between any LTSs (stable and unstable).

By Theorem 27, the congruences implied by " $\cong$ CFFD" that do not preserve initial stability are precisely the 40 congruences in Fig. 6. This result does not assume the congruence property with respect to infinite deterministic choice.

We now turn our attention to congruences that preserve initial stability. By Theorem 31 , they can be represented in the form " $\cong{ }_{y}^{x}$ ", where " $\cong y$ " can only be one of the 39 black or

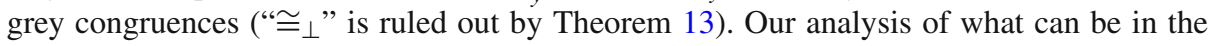
place of " $\cong_{x}$ " starts with the following observation.

Lemma 43 All black congruences in Fig. 6 have the property that if $L_{1}$ and $L_{2}$ are stable, then $L_{1} \cong L_{2}$ if and only if $\Sigma_{1}=\Sigma_{2}$, en $\left(L_{1}\right)=$ en $\left(L_{2}\right)$, and $a^{-1} L_{1} \cong a^{-1} L_{2}$ for every $a \in \operatorname{en}\left(L_{1}\right)$.

Proof Assume $L_{1} \cong L_{2}$. Any black congruence implies $\Sigma_{1}=\Sigma_{2}$. It also implies either $\operatorname{Tr}\left(L_{1}\right)=\operatorname{Tr}\left(L_{2}\right), \operatorname{Sf}\left(L_{1}\right)=\operatorname{Sf}\left(L_{2}\right)$, or $\operatorname{minD}\left(L_{1}\right)=\operatorname{minD}\left(L_{2}\right)$ and $\operatorname{anT}\left(L_{1}\right)=\operatorname{anT}\left(L_{2}\right)$. By Lemma 42 , en $\left(L_{1}\right)=\operatorname{en}\left(L_{2}\right)$ in all three cases. The definition of $a^{-1} L$ only uses operators with respect to which the congruence property was assumed. Therefore, $a^{-1} L_{1} \cong a^{-1} L_{2}$ for every $a \in \operatorname{en}\left(L_{1}\right)$.

We now prove the opposite direction. Every congruence in question has been defined via $\Sigma_{1}=\Sigma_{2}$ and $\mathrm{X}_{1}\left(L_{1}\right)=\mathrm{X}_{1}\left(L_{2}\right), \ldots, \mathrm{X}_{n}\left(L_{1}\right)=\mathrm{X}_{n}\left(L_{2}\right)$, where $\mathrm{X}_{1}, \ldots, \mathrm{X}_{n}$ are some sets in Fig. 6. We assume $\Sigma_{1}=\Sigma_{2}$, en $\left(L_{1}\right)=$ en $\left(L_{2}\right)$, and $\mathrm{X}_{i}\left(a^{-1} L_{1}\right)=\mathrm{X}_{i}\left(a^{-1} L_{2}\right)$ for every $1 \leq i \leq n$ and every $a \in \operatorname{en}\left(L_{1}\right)$, and have to prove $\Sigma_{1}=\Sigma_{2}$ and $\mathrm{X}_{1}\left(L_{1}\right)=\mathrm{X}_{1}\left(L_{2}\right), \ldots$, $\mathrm{X}_{n}\left(L_{1}\right)=\mathrm{X}_{n}\left(L_{2}\right)$.

Let $\mathrm{X}_{i}\left(a^{-1} L\right)$ denote the function that maps each $a \in \operatorname{en}(L)$ to $\mathrm{X}_{i}\left(a^{-1} L\right)$. Every set in Fig. 6 has the property that if $L$ is stable, then $\mathrm{X}_{i}(L)$ can be expressed as a function $f_{i}$ of en $(L)$ and $\mathrm{X}_{i}\left(a^{-1} L\right)$. For instance, $\operatorname{Tr}(L)=\{\varepsilon\} \cup\left\{a \sigma \mid a \in \operatorname{en}(L) \wedge \sigma \in \operatorname{Tr}\left(a^{-1} L\right)\right\}$ and $\operatorname{anF}(L)=\overline{\{(\varepsilon, A) \mid} A \cap \operatorname{en}(L)=\emptyset\} \cup\left\{(a \sigma, A) \mid a \in \operatorname{en}(L) \wedge(\sigma, A) \in \operatorname{anF}\left(a^{-1} L\right)\right\}$. Because $\mathrm{X}_{i}\left(a^{-1} L_{1}\right)=\mathrm{X}_{i}\left(a^{-1} L_{2}\right)$ for every $a \in \operatorname{en}\left(L_{1}\right)=\operatorname{en}\left(L_{2}\right)$, we have $\mathrm{X}_{i}\left(L_{1}\right)=$ $f_{i}\left(\mathrm{en}\left(L_{1}\right), \mathrm{X}_{i}\left(a^{-1} L_{1}\right)\right)=f_{i}\left(\mathrm{en}\left(L_{2}\right), \mathrm{X}_{i}\left(a^{-1} L_{2}\right)\right)=\mathrm{X}_{i}\left(L_{2}\right)$.

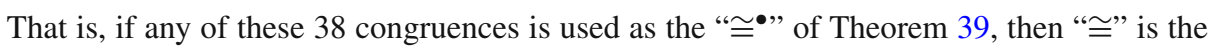
same congruence. The remaining two congruences compare at most the alphabets. For both

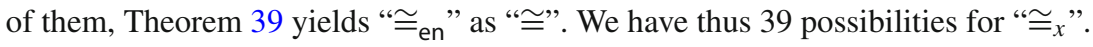


We have already argued that " $\cong \sum_{\sum}^{e n}$ " and the 38 " $\cong_{x}^{x}$ " are congruences with respect to the five operators in question. It remains to be shown that no combination of the " $\cong$ " and " $\cong y$ " found above yields an additional congruence.

Lemma 44 If " $\cong_{x}$ " is a black congruence, " $\cong_{y}$ " is a black or grey congruence, and " $\cong_{y}^{x}$ "

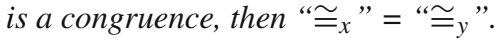

Proof Assume $L_{1} \cong{ }_{y} L_{2}$. By the congruence property, $\tau . L_{1} \cong y \tau . L_{2}$. By the definition of

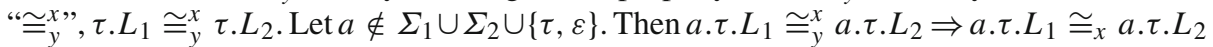
$\Rightarrow \tau \cdot \tau \cdot L_{1}=\left(a \cdot \tau \cdot L_{1}\right) \backslash\{a\} \cong_{x}\left(a \cdot \tau \cdot L_{2}\right) \backslash\{a\}=\tau \cdot \tau \cdot L_{2}$. Because $\tau \cdot L \cong_{\mathrm{CFFD}} L$ for any $L$, we have $L_{1} \cong_{\text {CFFD }} \tau \cdot \tau . L_{1}$ and $\tau . \tau . L_{2} \cong_{\text {CFFD }} L_{2}$. Because " $\cong_{\text {CFFD" implies }} \cong_{x}$, we have $L_{1} \cong{ }_{x} L_{2}$.

Assume $L_{1} \cong_{x} L_{2}$. Let $a \notin \Sigma_{1} \cup \Sigma_{2} \cup\{\tau, \varepsilon\}$. Then $a \cdot L_{1} \cong_{x} a \cdot L_{2} \Rightarrow a \cdot L_{1} \cong_{y}^{x} a \cdot L_{2}$

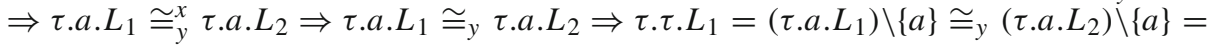

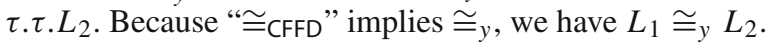

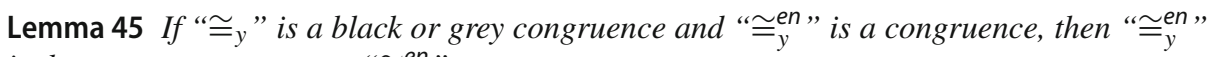

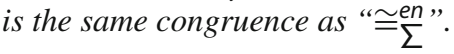

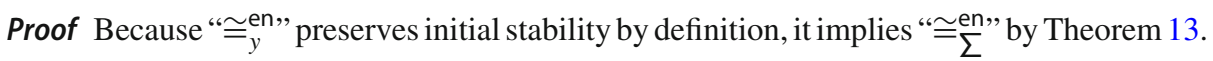

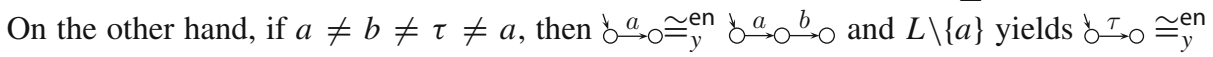
$\dot{b} \underset{\sim}{\rightarrow} \stackrel{b}{\rightarrow} 0$. This rules out the black congruences.

We have proven the following.

Theorem 46 The stability-preserving CFFD equivalence implies precisely 79 congruences with respect to parallel composition, hiding, relational renaming, action prefix, and infinite deterministic choice. They are the 40 congruences in Fig. 6, the 38 congruences of the form

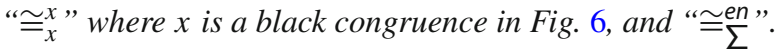

\section{Discussion}

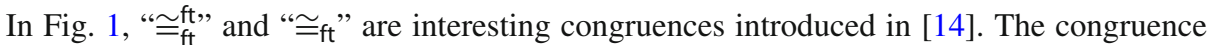
" $\cong_{\mathrm{tr}}$ " is the good old trace equivalence. " $\cong_{\mathrm{tr}}^{\mathrm{tr}}$ " is its obvious extension with stability. It seems unnecessary, because " $\cong$ tr" is a congruence with respect to the choice operator. The congruence " $\cong \Sigma$ " is trivial. Being the weakest stability-preserving congruence with respect to many widely used operators, " $\cong$ en" may have some interest. The remaining congruences of the form " $\simeq_{y}^{x}$ " feel artificial and go away in the presence of the action prefix operator, so they are probably unimportant.

The distinction of the eight congruences with the subscript \# or $\perp$ from the four congruences with the subscript $\Sigma$ is artificial, because it is a consequence of our choice of the parallel composition operator, which requires that each LTS has an alphabet of its own. We next comment on this decision.

Many authors use a global alphabet that is common to all LTSs. This convention needs a different parallel composition operator. A widely used option is $L_{1} \|_{A} L_{2}$, where $A$ is a set that does not contain $\tau$ or $\varepsilon$, and an action is executed jointly by $L_{1}$ and $L_{2}$ if and only if it is in $A$. If $a \notin A$ and both $L_{1}$ and $L_{2}$ can execute it, then they execute it one at a time.

The main reason for our convention is technical simplicity. Many of our constructions need actions that are not in the alphabets of any of the LTSs in question. With a global alphabet, an LTS may use all actions in it as labels of transitions, depriving us of outside actions. 
If the global alphabet is infinite, then actions can be liberated with a bijective renaming operator that maps the alphabet to its proper subset. However, this would be a complication in proofs that is not needed with our convention.

We now show that if the global alphabet consists of only one action, then there are additional congruences. Let $a$ be that action. If $L$ has arbitrarily long traces, then let $\operatorname{ml}(L):=\omega$. $\operatorname{Then} \operatorname{Tr}(L)=\{a\}^{*}$. Otherwise, let $\operatorname{ml}(L)$ denote the length of a longest trace of $L$. In this case, $\operatorname{Tr}(L)=\left\{a^{n} \mid n \leq \operatorname{ml}(L)\right\}$. For each $n \in \mathbb{N} \cup\{\omega\}$, the following is a congruence with respect to $\left\|_{\emptyset},\right\|_{\{a\}}$, and the six operators defined in Sect. 2: $L_{1} \cong L_{2}$ if and only if $\mathrm{ml}\left(L_{1}\right)=\mathrm{ml}\left(L_{2}\right) \leq n$ or $\mathrm{ml}\left(L_{1}\right) \geq n \leq \mathrm{ml}\left(L_{2}\right)$. This is an infinite sequence of distinct congruences between the trace equivalence (obtained with $n=\omega$ ) and the congruence that preserves nothing (obtained with $n=0$ ).

In conclusion, both our convention and the alternative introduce artificial congruences, but our convention simplifies the study of interesting congruences.

With our convention, any two LTSs with different alphabets have different tree failures for a vacuous reason. If $a \in \Sigma_{1}$ but $a \notin \Sigma_{2}$, then $L_{2}$ can neither execute nor refuse $a$, but either $a \in \operatorname{Tr}\left(L_{1}\right)$ or $(\varepsilon,\{a\}) \in \operatorname{Tf}\left(L_{1}\right)$. Our results on congruences that do not preserve the alphabet are not based on this trivial issue. Instead, Lemma 20 says that, roughly speaking, where the alphabet is not preserved, no information on traces is preserved. The congruence " $\cong$ tr " preserves some information on traces although it does not preserve the alphabet, but these happen with different classes of LTSs: stable with the former, and unstable with the latter.

In [17], all congruences implied by the not stability-preserving Chaos-Free Failures Divergences (CFFD) equivalence were found, assuming the congruence property with respect to parallel composition, hiding, relational renaming, and action prefix. Forty congruences were found (the CSP failures divergences equivalence being one of them). All but one of them preserve the alphabet. This is in sharp contrast with " $\cong$ ft", which implies only five congruences with respect to a strictly smaller set of operators.

In Sect. 5, we combined the requirement of initial stability with tree failures, traces, and alphabet preservation. To apply a similar strategy in the case of CFFD would require repeating the proofs in [17] also considering initial stability, which would be a huge amount of work (the publication contains 33 dense pages). Therefore, in Sect. 6 we developed a theory of dealing with initial stability as an add-on, and applied it in Sect. 7.

In Sect. 6, only the congruence property with respect to parallel composition and hiding was needed to prove that no new congruences arise that either do not preserve initial stability, or are used to compare unstable LTSs by a congruence that does preserve initial stability. This is a very general result. The comparison of stable LTSs when preserving initial stability proved much more difficult to deal with. As a consequence, we were unable to prove the existence or non-existence of congruences with a very weird property (congruences with respect to finite choice but not with respect to infinite deterministic choice) in the region below stability-preserving CFFD equivalence. Other than that, Theorem 46 fully analyses the region, finding 79 congruences.

A natural next topic would be to find all congruences that are implied by the intersection of " $\cong \mathrm{ft}$ " and CFFD equivalence, or less ambitiously, " $\cong \mathrm{ft}$ " and CFFD equivalence. The hard part is to find out whether there are congruences that are not intersections of those in [17] and the present study. Figure 1 may encourage to guess that this is impossible. However, [17] contains counter-examples, as seen in Fig. 6. For instance, the CSP failures divergences equivalence does not arise as an intersection of strictly weaker congruences in the figure. Sections 6 and 7 can be seen as generalizing results on " $\cong$ en" and CFFD equivalence to their intersection. Judging from the difficulties encountered and from the fact that initial stability 
is perhaps the simplest add-on one can think of, generalizing results on " $\cong_{\mathrm{ft}}$ " and CFFD equivalence to their intersection will perhaps not be trivial..

Acknowledgements Open access funding provided by University of Jyväskylä (JYU). The author thanks Walter Vogler for his comments on an early manuscript of the conference version of this study, and the reviewers of the conference version for careful reading and good suggestions for improvements. Also the reviewers of the journal version deserve great thanks for their hard work that led to many improvements to the presentation.

Open Access This article is licensed under a Creative Commons Attribution 4.0 International License, which permits use, sharing, adaptation, distribution and reproduction in any medium or format, as long as you give appropriate credit to the original author(s) and the source, provide a link to the Creative Commons licence, and indicate if changes were made. The images or other third party material in this article are included in the article's Creative Commons licence, unless indicated otherwise in a credit line to the material. If material is not included in the article's Creative Commons licence and your intended use is not permitted by statutory regulation or exceeds the permitted use, you will need to obtain permission directly from the copyright holder. To view a copy of this licence, visit http://creativecommons.org/licenses/by/4.0/.

\section{References}

1. Cormen, T.H., Leiserson, C.E., Rivest, R.L.: Introduction to Algorithms. MIT Press, Cambridge (1989)

2. De Nicola, R., Vaandrager, F.W.: Three logics for branching bisimulation. J. ACM 42(2), 458-487 (1995)

3. Emerson, E.A.: The beginning of model checking: a personal perspective. In: Grumberg, O., Veith, H. (eds.) 25 Years of Model Checking-History, Achievements, Perspectives. Lecture Notes in Computer Science, pp. 27-45. Springer, New York (2008)

4. Gazda M, Fokkink W (2010) Congruence from the operator's point of view: compositionality requirements on process semantics. In: Aceto, L., Sobocinski, P. (eds.) Proceedings Seventh Workshop on Structural Operational Semantics, SOS 2010, Paris, France, 30 August 2010, vol. 32, pp. 15-25. EPTCS

5. van Glabbeek, R.J.: The linear time—-branching time spectrum II. In: Best, E. (ed.) CONCUR '93, 4th International Conference on Concurrency Theory, Hildesheim, Germany, August 23-26, 1993, Proceedings, Volume 715 of Lecture Notes in Computer Science, pp. 66-81. Springer (1993)

6. van Glabbeek, R.J.: The coarsest precongruences respecting safety and liveness properties. In: Calude, C.S., Sassone, V. (eds.) Theoretical Computer Science-6th IFIP TC 1/WG 2.2 International Conference, TCS 2010, Held as Part of WCC 2010, Brisbane, Australia, September 20-23, 2010. Proceedings, Volume 323 of IFIP Advances in Information and Communication Technology, pp. 32-52. Springer (2010)

7. van Glabbeek, R.J., Luttik, B., Trcka, N.: Computation tree logic with deadlock detection. Logic. Methods Comput. Sci. 5(4:5), 1-24 (2009)

8. van Glabbeek, R.J., Weijland, W.P.: Branching time and abstraction in bisimulation semantics. J. ACM 43(3), 555-600 (1996)

9. Kaivola, R., Valmari, A.: The weakest compositional semantic equivalence preserving nexttime-less linear temporal logic. In: Cleaveland, R. (ed.) CONCUR '92, Third International Conference on Concurrency Theory, Stony Brook, NY, USA, August 24-27, 1992, Proceedings, Volume 630 of Lecture Notes in Computer Science, pp. 207-221. Springer (1992)

10. Milner, R.: Communication and Concurrency. PHI Series in Computer Science. Prentice Hall, Upper Saddle River (1989)

11. Puhakka, A.: Weakest congruence results concerning "'any-lock". In: Kobayashi, N., Pierce, B.C. (eds.) Theoretical Aspects of Computer Software, 4th International Symposium, TACS 2001, Sendai, Japan, October 29-31, 2001, Proceedings, Volume 2215 of Lecture Notes in Computer Science, pp. 400-419. Springer (2001)

12. Puhakka, A., Valmari, A.: Weakest-congruence results for livelock-preserving equivalences. In: Baeten, J.C.M., Mauw, S. (eds.) CONCUR '99: Concurrency Theory, 10th International Conference, Eindhoven, The Netherlands, August 24-27, 1999, Proceedings, Volume 1664 of Lecture Notes in Computer Science, pp. 510-524. Springer (1999)

13. Rabin, M.O.: Probabilistic algorithm for testing primality. J. Number Theory 12(1), 128-138 (1980)

14. Rensink, A., Vogler, W.: Fair testing. Inf. Comput. 205(2), 125-198 (2007)

15. Roscoe, A.W.: Understanding Concurrent Systems. Texts in Computer Science. Springer, New York (2010)

16. Valmari, A.: The weakest deadlock-preserving congruence. Inf. Process. Lett. 53(6), 341-346 (1995) 
17. Valmari, A.: All linear-time congruences for familiar operators. Logic. Methods Comput. Sci. 9(4:11), 1-34 (2013)

18. Valmari, A.: On constructibility and unconstructibility of LTS operators from other LTS operators. Acta Inf. 52(2-3), 207-234 (2015)

19. Valmari, A.: The congruences below fair testing with initial stability. In: Desel, J., Yakovlev, A. (eds.) 16th International Conference on Application of Concurrency to System Design, ACSD 2016, Torun, Poland, June 19-24, 2016, pp. 25-34. IEEE Computer Society (2016)

20. Valmari, A., Tienari, M.: Compositional failure-based semantics models for basic LOTOS. Formal Asp. Comput. 7(4), 440-468 (1995)

21. Valmari, A., Vogler, W.: Fair testing and stubborn sets. STTT 20(5), 589-610 (2018)

Publisher's Note Springer Nature remains neutral with regard to jurisdictional claims in published maps and institutional affiliations. 